\title{
Ecofriendly Synthesis in Aqueous Medium: An Expeditious Approach to New $N, N$-Diethyl Amide Bearing Benzenemethanesulfonamides
}

\author{
Olayinka O. Ajani*,1 Oluwole B. Familoni, ${ }^{2}$ Johnbull O. Echeme, ${ }^{1}$ and Feipeng $\mathrm{Wu}^{3}$ \\ ${ }^{1}$ Department of Chemistry, Covenant University, Canaanland, Km 10 Idiroko Road, P.M.B. 1023, Ota, Ogun State, \\ Nigeria \\ ${ }^{2}$ Department of Chemistry, University of Lagos, Akoka, Yaba, Lagos State, Nigeria \\ ${ }^{3}$ New Functional Polymeric Material Group, Technical Institute of Physics and Chemistry, Chinese Academy of Science \\ (CAS), Beijing 100190, P.R. China
}

\begin{abstract}
An highly expeditious synthetic approach for the synthesis of benzenemethanesulfonamides (1a-k) and their new corresponding $N, N$-diethyl substituted amido moieties (2a-k) has been achieved in aqueous medium at room temperature. The reaction condition was thoroughly optimized thereby allowing significant rate enhancement and resulting into excellent yields. The chemical structures of the successful candidates were confirmed using elemental analytical and spectroscopic data such as IR, ${ }^{1} \mathrm{H}$ NMR, ${ }^{13} \mathrm{C}$ NMR and some selected mass spectral data.
\end{abstract}

\section{Graphical Abstract}
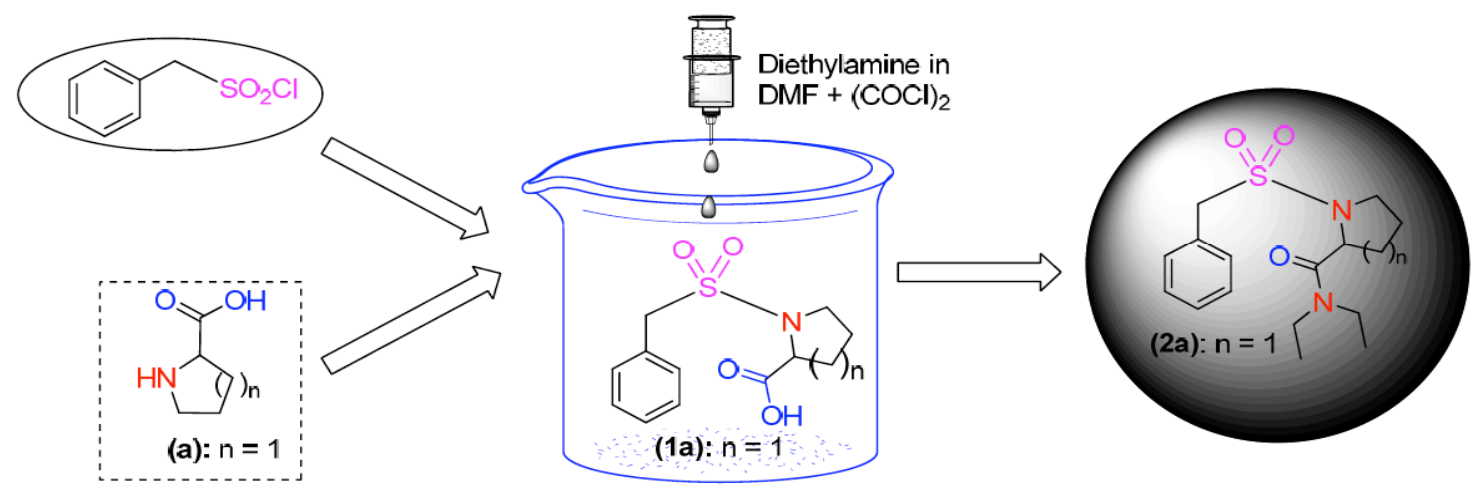

Environmental friendly synthesis of new $N, N$-diethyl-substituted-2-(phenylmethylsulfo namido)alkanamide derivatives was achieved in aqueous medium via synthetic modification of the benzenemethanesulfonamide precursors.

Keywords: sulfonamide, amino acids, spectroscopy, sulfonylation.

\section{INTRODUCTION}

Over the years, sulfonamides have been in clinical use [1, 2] and are known to represent a class of medicinally important compounds [3-5] which are extensively used as antibacterial agents [6-8]. Sulfonamide has played a historically fundamental role in the advancement of structure-activity relationships [9] and potential utility of pharmacokinetics in drug design [10]. Some currently approved drugs and recently reported drug-like entities with sulfonamide structural

*Address correspondence to this author at the Department of Chemistry, Covenant University, Canaanland, Km 10 Idiroko Road, P.M.B. 1023, Ota, Ogun State, Nigeria.; Tel: +2348061670254;

E-mails: ola.ajani@covenantuniversity.edu.ng; wajanfresh@yahoo.com skeletons are as shown in Fig. (1) below [4-7]. Although, arguably, the most direct route commonly used for synthesis of sulfonamides involves the nucleophilic attack of sulfonyl chloride by ammonia in the presence of a base [11]. However, it has also been reported that the condensation of sulfonyl chloride derivatives with various amino containing precursors such as ethanamine [12], propen-2-amine [13], cyclohexanamine [14], $p$-anisidine [15], benzo[d]thiazol-2amine [16], 8-aminoquinoline [17, 18], diprotected glutamic acid [19], pyrrole [20], substituted aniline [21], afforded the corresponding sulfonamide derivatives in encouraging yields.

Moreover, sulfonamides and their polymer-supported moieties are a common functional group $\left(-\mathrm{SO}_{2} \mathrm{NH}_{2}\right)$ in me- 


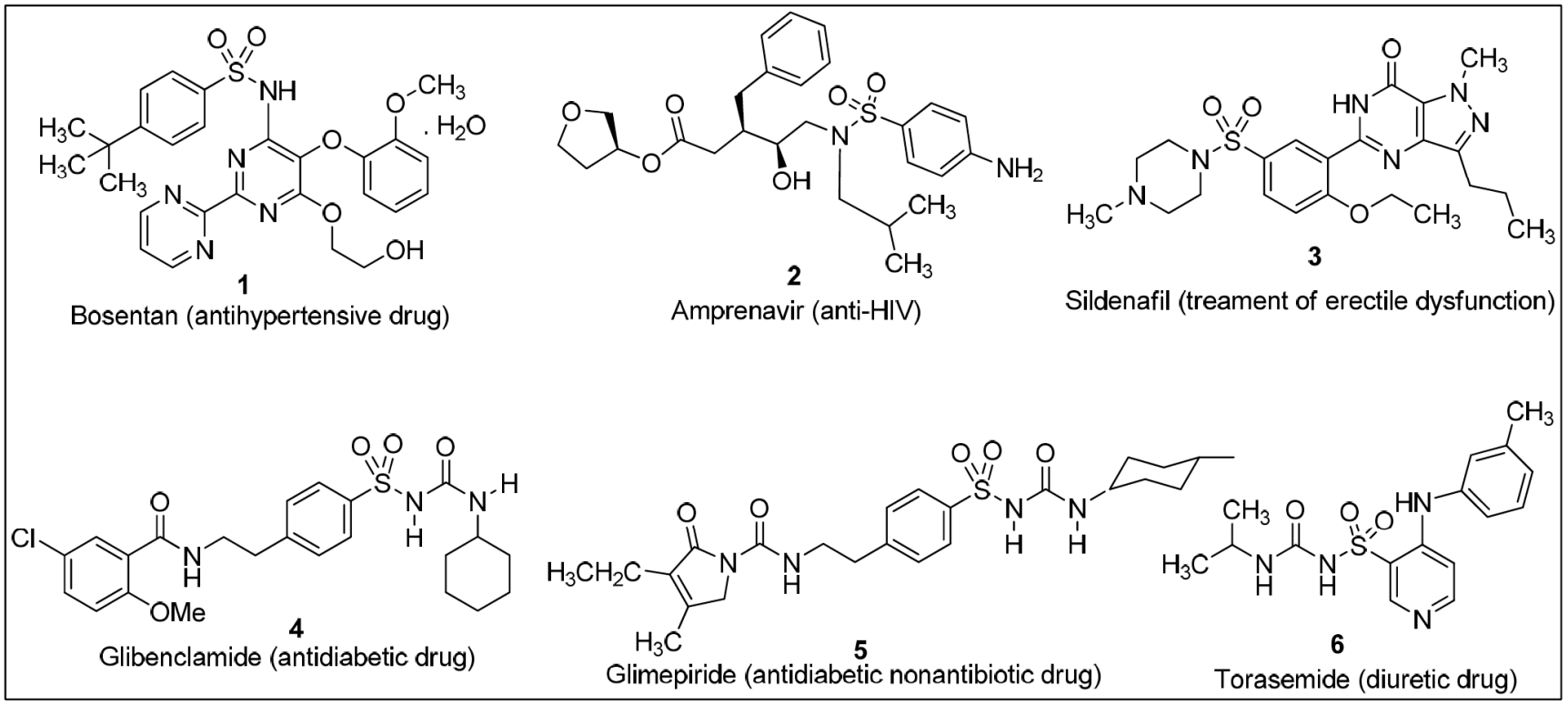

Fig. (1). Some important sulfonamide-based drugs available in market.

dicinal chemistry [22-24]. They have served in a variety of synthetic applications [25-27] which include preparation of polyazamacrocyclic compounds [28], asymmetric reduction of prochiral ketone [29, 30], ring-closing metathesis [31], bridged glycomimetics [32], carbanion-mediated sulfonamide intramolecular cyclizations [33], heterocyclic compounds synthesis [34], higher generation dendrimers [35] and Baylis-Hillman selective formation of $\alpha$-methylene- $\beta$ amino derivatives [36] among others. In addition, numerous reports have focused on benzenesulfonyl chloride and $p$ toluenesulfonyl chloride in the synthesis of bioactive sulfonamides [37-39]. Also, reaction of benzyl sulfonyl chloride with some amino acids had been reported to give sulfonamides but in low to poor yields [40]. This incident of poor yields calls for a facile approach to preparing sulfonamide in improved yields. Nevertheless, despite the wide knowledge garnered to date on sulfonylation, we noticed that there have so far been no effort toward preparation of $N, N$ diethyl substituted amide derivative of such benzenemethanesulfonamides except that of some benzenesulfonamides and $p$-tolylsulfonamides earlier reported [41].

Furthermore, toxicity of the reacting solvents and discharge of poisonous chemicals associated with synthesis of the common sulfonamide was herein overcome by using ecofriendly synthesis in aqueous medium driven in ice bath to room temperature. Based on the challenges aforementioned and compulsory need for environmental-friendly synthetic practices, it is conceivable to develop a highly efficient approach to synthesis of benzenemethanesulfonamides in better yields and to also achieve new $N, N$-diethyl substituted amide derivatives of such scaffolds in excellent yields.

\section{RESULTS AND DISCUSSION}

In many synthetic processes for preparing sulfonamide derivatives; not only byproducts, but also the reagents used for the production, may cause a threat to the environment. Synthesis at high thermal energy is also associated with frequent discharge of poisonous gases into the environment [42]. We have here-in described a successful approach for the synthesis of benzenemethanesulfonamides (1a-k) in high yields from the reaction of benzenemethanesulfonyl chloride with some readily available and cost effective L-amino acid precursors. The coupling reaction was initiated in ice bath and completed at ambient temperature in the presence of water as the molecular solvent of choice for environmentally-friendly synthetic approach. These benzenemethanesulfonamides have also been subsequently converted to $N, N$-diethyl alkanamide substituted scaffolds (2a-k) by benign protocol with an hope of producing benzothiazepine by metalation of the latter according to an earlier finding and reported procedure [41].

First and foremost, the coupling of benzenemethanesulfonyl chloride with eleven different amino acids resulted in the formation of the corresponding benzenemethanesulfonamide (1a-k) as shown in Scheme (1). Although, some of these benzenemethanesulfonamides (1a, $\mathbf{1 c}$ and $\mathbf{1 d})$ had been reported earlier in low yields [40], that is why it is very crucial to explore various reaction conditions for effective optimization study in order to establish another method to obtain these benzenemethanesulfonamides in high and encouraging yields without posing any threat to the environment. It is interesting to note that the $N, N$-diethyl amide bearing sulfonamides (2a-k) have not been reported before, to the best of our knowledge. So, preliminary optimization of reaction conditions was done by comparing the sulfonylation of Lproline as the representative of amino acids for the synthesis of (1a) under two different cases, namely: in the presence of (i) $\mathrm{Na}_{2} \mathrm{CO}_{3}$ base using water as the solvent (ii) triethylamine base using tetrahydrofuran (THF) solvent. It was discovered that although, in case ii, proline was effectively converted to its ammonium salt as a way of protecting the carboxyl functionality, but it failed to couple with benzenemethanesulfonyl chloride as envisaged. This was established through the ${ }^{1} \mathrm{H}$ NMR spectrum which had no aromatic-proton in the region around 6.0 to $8.0 \mathrm{ppm}$ downfield of tetramethylsilane 
(TMS) scale. Therefore, the case (ii) approach was discarded due to this disappointment.

On the other hand, case (i) condition using aqueous $\mathrm{Na}_{2} \mathrm{CO}_{3}$ gave the expected benzenemethanesulfonamide product named 1-(benzylsulfonyl) pyrrolidine-2-carboxylic acid (1a) via the modification of a known procedure [43]. Meanwhile, after establishing case (i) as the acceptable working procedure, it is thoughtful to play around thermodynamic potential in order to validate the best reaction temperature for maximum yield. Hence, the effect of variation in temperature was carefully studied by carrying out the coupling of L-proline with benzenemethanesulfonyl chloride in aqueous $\mathrm{Na}_{2} \mathrm{CO}_{3}$ at carefully controlled temperature of $0{ }^{\circ} \mathrm{C}$ to room temperature, at $60{ }^{\circ} \mathrm{C}$ to $80{ }^{\circ} \mathrm{C}$ and at $120{ }^{\circ} \mathrm{C}$ to 140 ${ }^{\circ} \mathrm{C}$ as shown in Entries 1, 2 and 3 respectively (Table 1). It was observed that the synthesis at $0{ }^{\circ} \mathrm{C}$ followed by stirring at room temperature afforded (1a) at excellent yield 92\%, while at an elevated temperature of $60{ }^{\circ} \mathrm{C}$ to $80{ }^{\circ} \mathrm{C}$, majority of the reacting species reverted back to the starting material thereby resulting in low (1a) yield of $37 \%$.

Unfortunately, at extremely high temperature $\left(120{ }^{\circ} \mathrm{C}\right.$ to $140{ }^{\circ} \mathrm{C}$ ) as shown in Entry 3 of Table 1, no meaningful isolable product was obtained. This was because of thermal decomposition of the L-proline precursor experienced at this unfriendly reaction temperature. Based on this result, it could be seen that the best optimization condition is to couple Lproline with benzenemethanesulfonyl chloride in aqueous $\mathrm{Na}_{2} \mathrm{CO}_{3}$ at $0{ }^{\circ} \mathrm{C}$ to room temperature. This successfully established procedure was then repeated for ten other amino acids to get the corresponding benzenemethanesulfonamides (1bk) in good to excellent yields. Difficulty connected with the poor reactivity of the benzenemethanesulfonyl chloride was overcome by increasing the duration of stirring at room temperature to $48 \mathrm{~h}$. The efficiency of the method used here-in (X) over the earlier reported approach (Y) [40] was evaluated via comparative study as shown in Table $\mathbf{2}$. For instance, the synthesis of (1a) earlier reported was achieved in $22 \%$ yield whereas excellent augmentation was experienced in our study here-in, which afforded (1a) at $92 \%$ yield. The result in Table 2 revealed that the method used in this study provided an elegant pathway towards synthesis of benzenemethanesufonamide (1a-k) in excellent yields.

It was observed that the work-up process to get the benzenemethanesulfonamides (1a-k) in solid form from acidified aqueous medium was very difficult due to high polarity of such sulfonamides unlike the benzenesulfonamide and $p$ tolylsulfonamide which normally crystallized out easily after acidification. This arbitrary solubility trend in benzenemethanesulfonamide is a strong indication that insertion of a sp ${ }^{3}$ hybridized carbon between the phenyl and $\mathrm{SO}_{2}$ unit

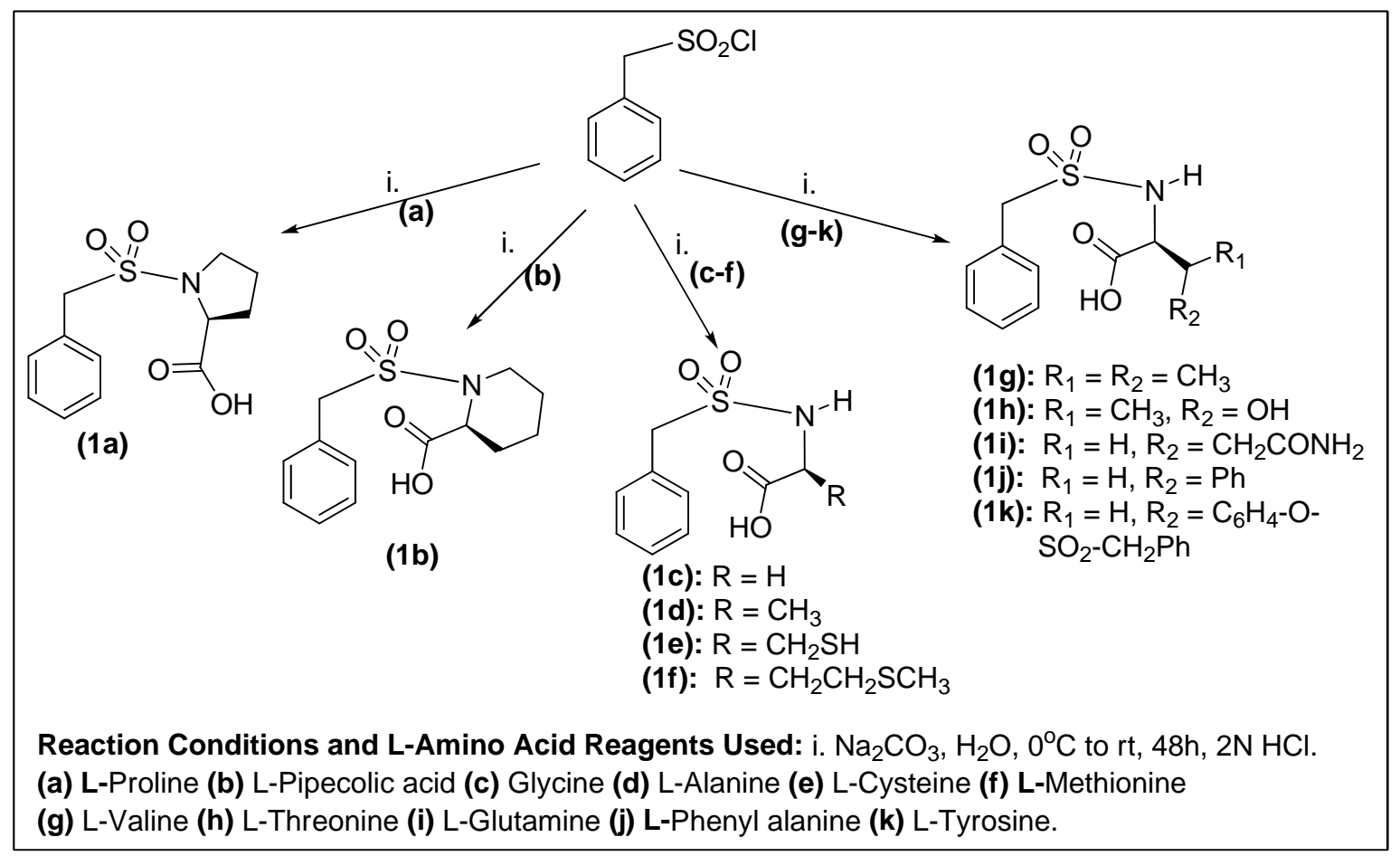

Scheme (1). Synthesis of benzenemethanesulfonamide derivatives (1a-k).

Table 1. Synthesis of (1a) at Different Temperatures for Optimization Study

\begin{tabular}{|c|c|c|c|c|}
\hline Entry & Reagent/Solvent & Temperature ${ }^{\mathbf{}} \mathbf{C}$ & Time h & 48 \\
\hline \hline 1 & $\mathrm{Na}_{2} \mathrm{CO}_{3} / \mathrm{H}_{2} \mathrm{O}$ & 0 to rt & 48 & 92 \\
\hline 2 & $\mathrm{Na}_{2} \mathrm{CO}_{3} / \mathrm{H}_{2} \mathrm{O}$ & $60-80$ & 48 & 48 \\
\hline 3 & $\mathrm{Na}_{2} \mathrm{CO}_{3} / \mathrm{H}_{2} \mathrm{O}$ & $120-140$ & - \\
\hline
\end{tabular}


Table 2. Comparative Study of Current and Reported Method for Synthesis of (1a-k)

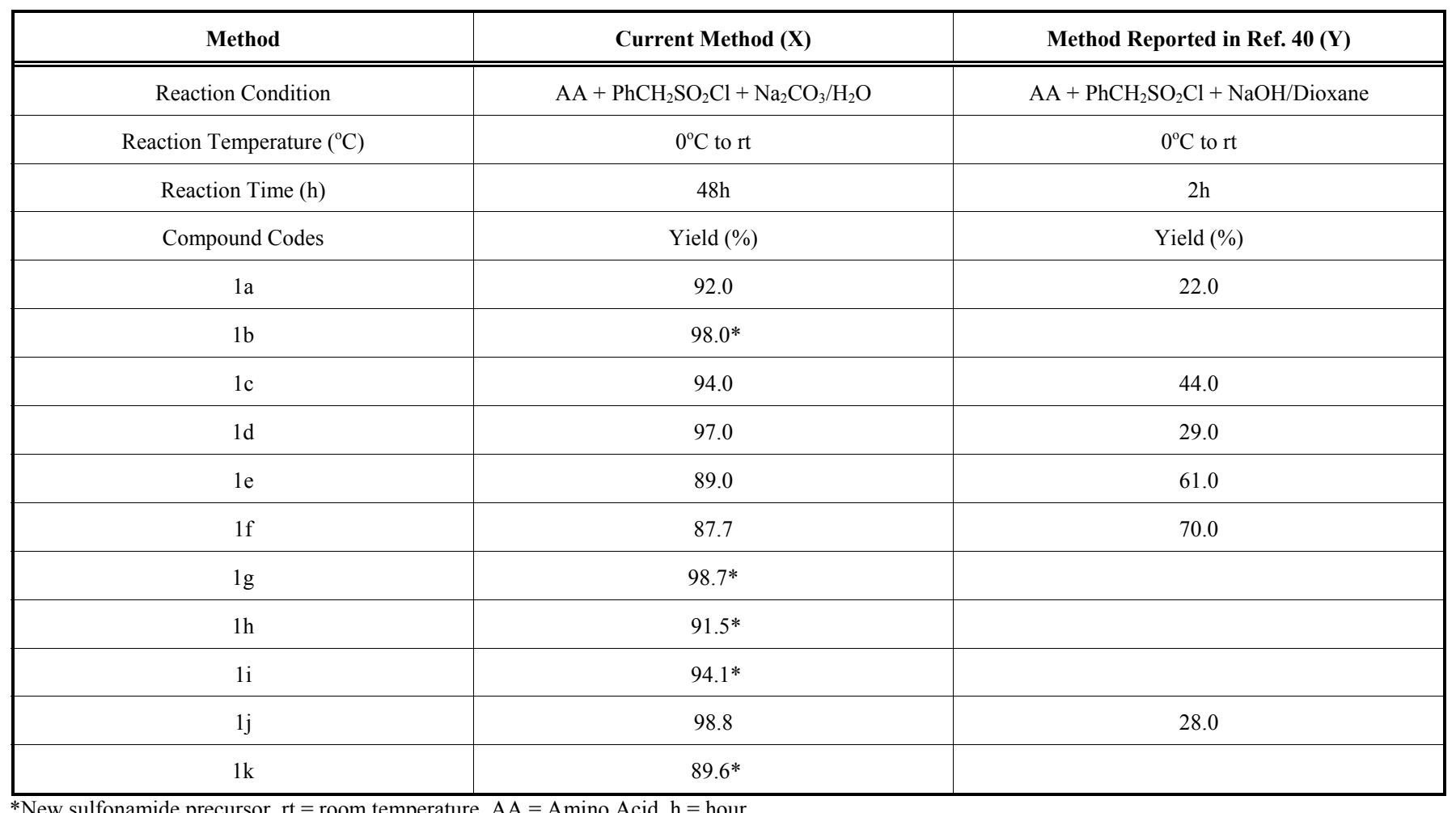

New sulfonamide precursor, $\mathrm{rt}=$ room temperature, $\mathrm{AA}=$ Amino Acid, $\mathrm{h}=$ hour

(i.e. $\mathrm{Ph}-\mathrm{CH}_{2}-\mathrm{SO}_{2} \mathrm{Cl}$ ) confers different behaviour on the benzenemethanesulfonamide, contrary to that of the common sulfonamides such as benzenesulfonamide and $p$ tolylsulfonamide. This challenge was overcome in this present study by using lyophilization technique to finalize the work-up process, followed by purification of the bulk solid products via column chromatography chloroform/methanol (3:1) as the eluting solvent to obtain pure compounds (1a-k).

The structures of all these compounds (1a-k) were confirmed using FT-IR, ${ }^{1} \mathrm{H}$ and ${ }^{13} \mathrm{C}$ NMR, mass spectral and elemental analytical data. In a general term, IR spectra of compounds (1a-k) showed absorption bands due to the stretching vibrations of $\mathrm{O}-\mathrm{H}$ of acid, $\mathrm{CH}$ aromatic, $\mathrm{CH}$ aliphatic, $\mathrm{C}=\mathrm{O}$ and $\mathrm{C}=\mathrm{C}$ at $3447-3404 \mathrm{~cm}^{-1}, 2990-2974 \mathrm{~cm}^{-1}$, $2878-2774 \mathrm{~cm}^{-1}, 1751-1728 \mathrm{~cm}^{-1}$ and $1620-1590 \mathrm{~cm}^{-1}$ respectively. They all experienced stretching vibrational frequency of $\mathrm{SO}_{2}$ units as two bands near 1238-1206 $\mathrm{cm}^{-1}$ and 1171$1151 \mathrm{~cm}^{-1}$ whereas Ar-H bending vibrational modes were noticed around $810-698 \mathrm{~cm}^{-1}$ as expected. In particular, the IR spectrum of (1a) as a representative of this group, exhibited the absorption band at $3441 \mathrm{~cm}^{-1}$ due to the presence of $\mathrm{O}-\mathrm{H}$ of carboxylic acid while bands for the $\mathrm{CH}$ of both aromatic and aliphatic were noticed at $2980 \mathrm{~cm}^{-1}$ and $2828 \mathrm{~cm}^{-1}$ respectively. Also, the stretching vibrational bands at 1728 $\mathrm{cm}^{-1}$ and $1620 \mathrm{~cm}^{-1}$ depicted $v(\mathrm{C}=\mathrm{O})$ of acid and $v(\mathrm{C}=\mathrm{C})$ of aromatic respectively while the two bands at $1219 \mathrm{~cm}^{-1}$ and $1151 \mathrm{~cm}^{-1}$ were as a result of the presence of $\mathrm{SO}_{2}$ functionality. The mass spectral data of (1a) showed the molecular ion peak at $\mathrm{m} / \mathrm{z} 269.1$ which correlated well with the molecular mass of the compound (269.3) while the base peak found at $\mathrm{m} / \mathrm{z} 178.1$ was as a result of loss of benzylic radical $\left(\mathrm{PhCH}_{2}\right)$. Other daughter peaks produced by some fragmen- tation patterns of (1a), were observed at $\mathrm{m} / \mathrm{z}$ 179.1, 176.1, 122.0 and 105.0 with relative intensities of $18.4 \%, 32.4 \%$, $49 \%$ and $32 \%$ respectively.

In addition, the chemical shifts and the multiplicity patterns of ${ }^{1} \mathrm{H}$ and ${ }^{13} \mathrm{C}$ NMR were consistent with that of the proposed structure for all the sulfonamides of which (1a) is the targeted representative. So, the ${ }^{1} \mathrm{H}$ NMR spectrum of (1a) in $\mathrm{D}_{2} \mathrm{O}$ exhibited a five aromatic protons singlet at $\delta 7.47$ and two protons singlet of benzylic methylene (i.e. $\mathrm{Ph}-\mathrm{CH}_{2}-$ $\left.\mathrm{SO}_{2}\right)$ at $\delta$ 4.24. All other signals observed at upfield of TMS from $\delta 3.48-3.45$ to $\delta 2.15-2.09$ were due to the presence of six pyrrolo protons while that of the seventh pyrrolo proton ( $\mathrm{CH}-\mathrm{COO})$ resonated as a doublet of doublet at $\delta$ 4.46-4.43. Furthermore, ${ }^{13} \mathrm{C}$ NMR spectrum of (1a) ran in $\mathrm{D}_{2} \mathrm{O}$ in the presence of a few drops of dioxane solvent showed the presence of twelve different carbon atoms with the signals ranging from $173(\mathrm{C}=\mathrm{O})$ to $24.3\left(\mathrm{CH}_{2}\right) \mathrm{ppm}$.

Secondly, the conversion of benzenemethanesulfonamide (1a) (i.e. 1-(benzyl sulfonyl) pyrrolidine-2-carboxylic acid) to the targeted novel corresponding $N, N$-diethyl alkanamide substituted benzenemethanesulfonamide (2a) (i.e. 1(benzylsulfonyl)- $N, N$-diethyl pyrrolidine-2-carboxamide) was achieved via one-pot two-step amidation of the former via the modification of a known procedure [44]. In more detail, hence, (2a-k) were prepared by reacting the appropriate benzenemethanesulfonamides (1a-k) with oxalyl chloride in the presence of catalytic amount of DMF for the generation of acid chloride which was subsequently treated with diethyl amine in the presence of $\mathrm{Na}_{2} \mathrm{CO}_{3}$ in aqueous medium to afford (2a-k) in excellent yield (Scheme 2). The result of the physical parameters in terms of the molecular weights, melting points, $R_{f}$ values as well as the percentage yields of 


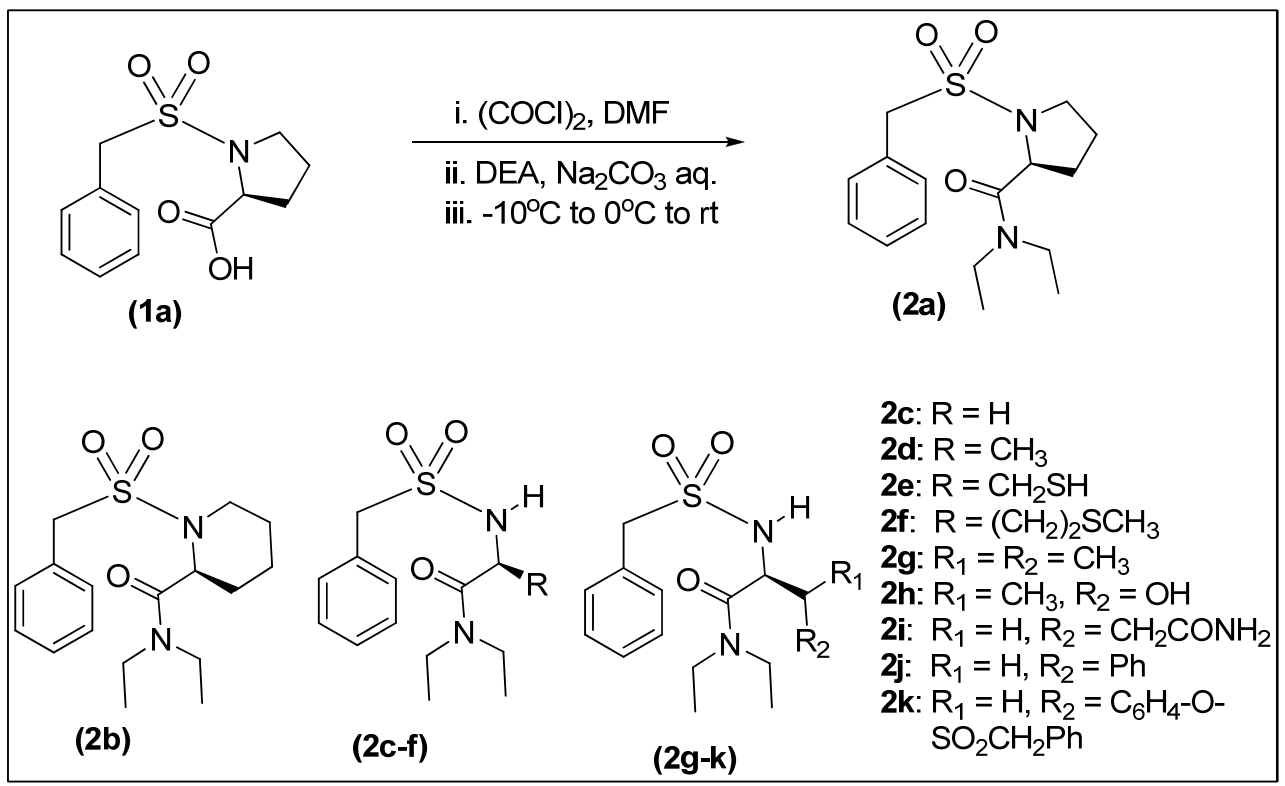

Scheme (2). Synthesis of $N, N$-diethylamido substituted benzenemethanesulfonamide (2a-k).

Table 3. Physical Data of $N, N$-diethyl Substituted Benzenemethanesulfonamides (2a-k)

\begin{tabular}{|c|c|c|c|c|c|c|c|}
\hline Sample Code & $\mathbf{R}$ & $\mathbf{R}_{1}$ & $\mathbf{R}_{\mathbf{2}}$ & Molecular Weight & $\mathrm{Mp}{ }^{\circ} \mathrm{C}$ & $\mathbf{R}_{\mathrm{f}}^{\mathrm{a}}$ & Yield \\
\hline (2a) & - & - & - & 324.45 & $185-187$ & 0.71 & 97.9 \\
\hline (2b) & - & - & - & 338.47 & $210-211$ & 0.72 & 99.0 \\
\hline$(2 \mathrm{c})$ & $\mathrm{H}$ & - & - & 284.38 & $213-215$ & 0.51 & 92.6 \\
\hline$(2 \mathrm{~d})$ & $\mathrm{CH}_{3}$ & - & - & 298.41 & $238-240$ & 0.56 & 98.5 \\
\hline$(2 \mathrm{e})$ & $\mathrm{CH}_{2} \mathrm{SH}$ & - & - & 330.47 & $198-200$ & 0.71 & 89.0 \\
\hline$(2 \mathrm{f})$ & $\left(\mathrm{CH}_{2}\right)_{2} \mathrm{SCH}_{3}$ & - & - & 358.53 & $170-172$ & 0.65 & 90.6 \\
\hline$(2 \mathrm{~g})$ & - & $\mathrm{CH}_{3}$ & $\mathrm{CH}_{3}$ & 326.46 & $226-230$ & 0.69 & 97.3 \\
\hline (2h) & - & $\mathrm{CH}_{3}$ & $\mathrm{OH}$ & 328.43 & $240(\mathrm{dec})$ & 0.53 & 80.2 \\
\hline (2i) & - & $\mathrm{H}$ & $\mathrm{CH}_{2} \mathrm{CONH}_{2}$ & 355.46 & $251-253$ & 0.58 & 93.2 \\
\hline$(2 \mathrm{j})$ & - & $\mathrm{H}$ & $\mathrm{Ph}$ & 374.51 & $227-229$ & 0.70 & 90.2 \\
\hline$(2 \mathrm{k})$ & - & $\mathrm{H}$ & OTs & 544.69 & $265(\mathrm{dec})$ & 0.69 & 89.3 \\
\hline
\end{tabular}

${ }^{a}$ Solvent system. Chloroform: Methanol (3:1)

the synthesized $N, N$-diethyl alkanamide substituted benzenemethanesulfonamides (2a-k) is as shown in Table $\mathbf{3}$.

Hence, the molecular weights of (2a-k) ranged from 284.38 to 544.69 while the melting points ranged from 170 $172{ }^{\circ} \mathrm{C}$ to $251-253{ }^{\circ} \mathrm{C}$ except that of (2h) and (2k) which decomposed at $240{ }^{\circ} \mathrm{C}$ and $265{ }^{\circ} \mathrm{C}$ respectively. It was also observed that upon TLC spotting, each reaction gave one spot each in chloroform: methanol (3:1) solvent system with the $R_{f}$ values varying between 0.51 and 0.72 . From the high polar nature of the solvent system and the $\mathrm{R}_{\mathrm{f}}$ values obtained, it could be easily seen that these compounds were very polar. No wonder, they were completely soluble in water and refused to crystallize even after several days. Hence, this justified why the only method by which the solid (2a-k) were gotten out of the solution was lyophilization technique. It was highly motivating to see that the entire solid (2a-k) were obtained in excellent yields ranging between a minimum of $80.2 \%$ for (2h) to a maximum of $99.0 \%$ for (2b) according to Table 3.

The spectroscopic properties of the synthesized $\mathrm{N}, \mathrm{N}$ diethylalkanamide substituted benzenemethanesulfonamides (2a-k) were studied and carefully examined for the structural characterization using IR, ${ }^{1} \mathrm{H}$ and ${ }^{13} \mathrm{C}$ NMR and mass spectral data. It was discovered that all the spectroscopic parameters were consistent with the proposed structures for (2a-k) (see experimental).

\section{EXPERIMENTAL}

\section{General Methods}

The melting points were determined on X-4 Digital Microscopic melting point apparatus and were uncorrected. The 
${ }^{1} \mathrm{H}$ nuclear magnetic resonance (NMR) spectra were recorded in $\mathrm{D}_{2} \mathrm{O}$ on NMR Bruker DPX 400 spectrometer operating at $400 \mathrm{MHz}$. Tetramethylsilane (TMS) was used as internal standard with the deuterium signal of the solvent as the lock and chemical shifts $\delta$ recorded in ppm. The ${ }^{13} \mathrm{C}$ NMR spectra were run in $\mathrm{D}_{2} \mathrm{O}$ being the only solubilizing solvent for these sulfonamides, but in the presence of one drop of Dioxane at $100 \mathrm{MHz}$ frequency since $\mathrm{D}_{2} \mathrm{O}$ had no carbon atom. Infrared (IR) spectra were run on Varian Excalibur HE 3100 FT-IR Spectrometer while the Mass Spectra (MS) were obtained using Waters GCT Premier Spectrometer. The elemental analysis $(\mathrm{C}, \mathrm{H}, \mathrm{N})$ of the compounds were performed using Flash EA 1112 Elemental Analyzer. Lyophilization was carried out where necessary by using FD-1 Freeze Drier while concentration and removal of solvents was achieved with RE-2000B Buchi Rotary Evaporator. The $\mathrm{pH}$ was monitored using Portable $\mathrm{pH}$ Meter Model PHB4 (accurate to $\pm 0.01 \mathrm{pH}$ units) while all drying were conducted at reduced pressure with DHG-9023A Vacuum Oven. The reaction progress was monitored with TLC using $\mathrm{CHCl}_{3} / \mathrm{CH}_{3} \mathrm{OH}$ (3:1) solvent system and the developed plates were visualized under UV lamp and/or in iodine tank where necessary. Column chromatographic purifications were carried out on Merck silica gel F (mesh 200-300). Oxalyl chloride and phenylmethylsulfonyl chloride were obtained from Alfa Aesar Chemicals while all other reagents were obtained from Beijing Chemical Works, China.

\section{Procedure for Synthesis of Benzenemethanesulfonamides} (1)

To a solution of amino acid $(5 \mathrm{mmol})$ in $\mathrm{H}_{2} \mathrm{O}(6 \mathrm{~mL})$ was added $\mathrm{Na}_{2} \mathrm{CO}_{3}(1.113 \mathrm{~g}, 10.5 \mathrm{mmol})$ with continuous stirring until all the solutes had dissolved. The clear solution was cooled to $-10{ }^{\circ} \mathrm{C}$ and benzenemethanesulfonyl chloride $(1.144 \mathrm{~g}, 6 \mathrm{mmol})$ was added in three batches over a period of $1 \mathrm{~h}$. It was warmed up to $0{ }^{\circ} \mathrm{C}$ and stirred there for $1 \mathrm{~h}$. Finally, the reacting mixture was then warmed up to room temperature and allowed to stir there for $48 \mathrm{~h}$. The reaction was quenched by addition of dichloromethane DCM $(10 \mathrm{~mL})$ and transferred into separatory funnel where the excess of benzenemethanesulfonyl chloride was removed by extraction. The aqueous layer was then worked up to give a clear solution by addition of $2 \mathrm{~N} \mathrm{HCl}$ until the $\mathrm{pH} 2.2$ was attained. The clear liquid was then lyophilized at $-52{ }^{\circ} \mathrm{C}$ under reduced pressure for $12 \mathrm{~h}$ to obtain the crude solid product which was purified by column chromatography $\left(\mathrm{CHCl}_{3} / \mathrm{CH}_{3} \mathrm{OH}, 3: 1\right)$ to afford benzenemethanesulfonamides (1) in good to excellent yields.

1-(Benzylsulfonyl)pyrrolidine-2-carboxylic acid (1a): Yield $1.24 \mathrm{~g}(92 \%) ; \mathrm{mp} 108-110{ }^{\circ} \mathrm{C}\left\{\right.$ Lit. $\left.107-108{ }^{\circ} \mathrm{C}\right\}[40]$; $\mathrm{R}_{\mathrm{f}}=0.84 ;{ }^{1} \mathrm{H}$ NMR $\left(\mathrm{D}_{2} \mathrm{O}, 400 \mathrm{MHz}\right) \delta: 7.47(\mathrm{~s}, 5 \mathrm{H}, \mathrm{Ar}-\mathrm{H})$, 4.46-4.43 (dd, $J_{1}=7.2 \mathrm{~Hz}, J_{2}=15.76 \mathrm{~Hz}, 1 \mathrm{H}, \mathrm{HOOC}-\mathrm{CH}-$ $\left.\mathrm{CH}_{2}(\mathrm{a}, \mathrm{b})\right), 4.24$ (s, $\left.2 \mathrm{H}, \mathrm{CH}_{2}-\mathrm{SO}_{2}\right), 3.48-3.45(\mathrm{t}, J=7.28 \mathrm{~Hz}$, $\left.2 \mathrm{H}, \mathrm{N}-\mathrm{CH}_{2}-\mathrm{CH}_{2}\right), 2.46-2.45\left(\mathrm{~m}, 1 \mathrm{H}, \mathrm{CHa}\right.$ of $\left.\mathrm{CH}_{2}\right), 2.22-2.21$ $\left(\mathrm{m}, 1 \mathrm{H}, \mathrm{CHb}\right.$ of $\mathrm{CH}_{2}$ ) , 2.15-2.09 (quintet, $J=6.8 \mathrm{~Hz}, 2 \mathrm{H}$, $\left.\mathrm{CH}_{2}-\mathrm{CH}_{2}-\mathrm{CH}_{2}(\mathrm{a}, \mathrm{b})\right) \mathrm{ppm} ;{ }^{13} \mathrm{C}$ NMR (Dioxane, $\left.100 \mathrm{MHz}\right) \delta$ : $173.1(\mathrm{CO}), 132.6,131.2$ (2CH aromatic), $129.5(2 \mathrm{CH}$ aromatic), 128.9, 60.6, 57.7, 47.1, 29.2, 24.3 ppm; IR (KBr) v: 3441 (OH of acid), 2980 ( $\mathrm{CH}$ aromatic), $2828(\mathrm{CH}$ aliphatic), $1728(\mathrm{C}=\mathrm{O}$ of $\mathrm{COOH}), 1620(\mathrm{C}=\mathrm{C}), 1219,1151$ ( $\mathrm{SO}_{2}$ two bands), $700(\mathrm{Ar}-\mathrm{H}) \mathrm{cm}^{-1}$; MS: in $\mathrm{m} / \mathrm{z}$ [rel. \%]:
$270.1\left[\mathrm{MH}^{+}, 6.5 \%\right], 269.1\left[\mathrm{M}^{+}, 9 \%\right], 179.1[18.4 \%], 178.1$ $\left[\mathrm{M}^{+}-\mathrm{PhCH}_{2}, 100 \%\right], 176.1$ [32.4\%], 122.0 [49\%], 105.0 [32\%]. Anal caled for $\mathrm{C}_{12} \mathrm{H}_{15} \mathrm{NO}_{4} \mathrm{~S}$ (269.32): C 53.52, H 5.61, N 5.20; found: C 53.67, H 5.49, N 5.34.

1-(Benzylsulfonyl)piperidine-2-carboxylic acid (1b): Yield $1.38 \mathrm{~g}(98 \%) ; \mathrm{mp} 248{ }^{\circ} \mathrm{C}(\mathrm{dec}) ; \mathrm{R}_{\mathrm{f}}=0.87 ;{ }^{1} \mathrm{H}$ NMR $\left(\mathrm{D}_{2} \mathrm{O}, 400 \mathrm{MHz}\right) \delta: 7.52(\mathrm{~s}, 5 \mathrm{H}, \mathrm{Ar}-\mathrm{H}), 4.28\left(\mathrm{~s}, 2 \mathrm{H}, \mathrm{CH}_{2-}\right.$ $\left.\mathrm{SO}_{2}\right), 4.08-4.03\left(\mathrm{dd}, J_{1}=3.44 \mathrm{~Hz}, J_{2}=15.12 \mathrm{~Hz}, 1 \mathrm{H}\right.$, HOOC- $\left.\mathrm{CH}-\mathrm{CH}_{2}\right), 3.57-3.53\left(\mathrm{~m}, 1 \mathrm{H}, \mathrm{CHa}\right.$ of $\left.\mathrm{CH}_{2}-\mathrm{N}\right), 3.16-$ $3.10\left(\mathrm{~m}, 1 \mathrm{H}, \mathrm{CHb}\right.$ of $\left.\mathrm{CH}_{2}-\mathrm{N}\right), 2.40-2.36(\mathrm{~m}, 1 \mathrm{H}, \mathrm{CH}), 2.01-$ $1.92(\mathrm{~m}, 2 \mathrm{H}, 2 \times \mathrm{CH}), 1.81-1.68\left(\mathrm{~m}, 3 \mathrm{H}, \mathrm{CH} \& \mathrm{CH}_{2}\right) \mathrm{ppm}$; ${ }^{13} \mathrm{C}$ NMR (Dioxane, $\left.100 \mathrm{MHz}\right) \delta: 172.5$ (CO), 132.5, 131.2 (2CH aromatic), 129.5 (2CH aromatic), 128.9, 57.7, 57.6, 44.8, 26.5, $22.1\left(2 \mathrm{CH}_{2}\right) \mathrm{ppm}$; IR ( $\left.\mathrm{KBr}\right)$ v: 3422 ( $\mathrm{OH}$ of acid), 2974 ( $\mathrm{CH}$ aromatic), 2822 ( $\mathrm{CH}$ aliphatic), $1736(\mathrm{C}=\mathrm{O}$ of $\mathrm{COOH}), 1603(\mathrm{C}=\mathrm{C}), 1238,1159\left(\mathrm{SO}_{2}\right.$ two bands $), 700(\mathrm{Ar}-$ H) $\mathrm{cm}^{-1}$; MS: in m/z [rel. \%]: $269.1\left[\mathrm{M}^{+}-\mathrm{CH}_{2}{ }^{\circ}, 3.2 \%\right], 180.1$ [55\%], 179.1 [65\%], 178.1 [M $\left.\mathrm{M}^{+}-\mathrm{PhCH}_{2}, 100 \%\right], 165.1$ [30\%], 121.0 [42\%], $77.0\left[\mathrm{Ph}^{+}, 13 \%\right], 64.0\left[\mathrm{SO}_{2}{ }^{+}, 31.7 \%\right]$. Anal calcd for $\mathrm{C}_{13} \mathrm{H}_{17} \mathrm{NO}_{4} \mathrm{~S}$ (283.35): C $55.11, \mathrm{H} 6.05, \mathrm{~N}$ 4.94; found: C 55.29, H 5.94, N 4.86.

2-(Phenylmethylsulfonamido)acetic acid (1c): Yield 1.08 g (94\%); mp $150-151{ }^{\circ} \mathrm{C}\left\{\right.$ Lit. $\left.151-152{ }^{\circ} \mathrm{C}\right\}[40] ; \mathrm{R}_{\mathrm{f}}=$ $0.51 ;{ }^{1} \mathrm{H}$ NMR $\left(\mathrm{D}_{2} \mathrm{O}, 400 \mathrm{MHz}\right) \delta: 7.45(\mathrm{~s}, 5 \mathrm{H}, \mathrm{Ar}-\mathrm{H}), 4.19$ (s, 2H, $\mathrm{CH}_{2}-\mathrm{SO}_{2}$ ), 3.76 (s, 2H, $\left.\mathrm{CH}_{2}-\mathrm{COOH}\right) \mathrm{ppm} ;{ }^{13} \mathrm{C} \mathrm{NMR}$ (Dioxane, $100 \mathrm{MHz}) \delta$ : $170.6(\mathrm{CO}), 132.7,131.2(2 \mathrm{CH}$ aromatic), 129.5 (2CH aromatic), 128.9, 57.7, $40.8 \mathrm{ppm}$; IR $(\mathrm{KBr})$ v: $3433(\mathrm{OH}$ of acid), $3030(\mathrm{~N}-\mathrm{H}), 2990(\mathrm{CH}$ aromatic), 2832 ( $\mathrm{CH}$ aliphatic), $1736(\mathrm{C}=\mathrm{O}$ of $\mathrm{COOH}), 1616$ $(\mathrm{C}=\mathrm{C}), 1215,1171\left(\mathrm{SO}_{2}\right.$ two bands), $702(\mathrm{Ar}-\mathrm{H}) \mathrm{cm}^{-1}$; MS: in $\mathrm{m} / \mathrm{z}$ [rel. \%]: $212.1\left[\mathrm{M}^{+}-\mathrm{OH}, 7.9 \%\right.$ ], 180.1 [73\%], 179.1 [88\%], $178.1\left[\mathrm{M}^{+}-\mathrm{PhCH}_{2}, 100 \%\right], 91.1\left[\mathrm{PhCH}_{2}^{+}, 48 \%\right], 64$ [26\%] $45\left[{ }^{+} \mathrm{COOH}, 2.4 \%\right]$. Anal calcd for $\mathrm{C}_{9} \mathrm{H}_{11} \mathrm{NO}_{4} \mathrm{~S}$ (229.26): C 47.15, H 4.84, N 6.11; found: C 46.99, H 5.01, N 5.97 .

2-(Phenylmethylsulfonamido)propanoic acid (1d): Yield 1.18 g (97\%); mp $126-128^{\circ} \mathrm{C}$ \{Lit. $127-128{ }^{\circ} \mathrm{C}$ \} [40]; $\mathrm{R}_{\mathrm{f}}=0.81 ;{ }^{1} \mathrm{H}$ NMR $\left(\mathrm{D}_{2} \mathrm{O}, 400 \mathrm{MHz}\right) \delta: 7.47(\mathrm{~s}, 5 \mathrm{H}$, Ar-H), $4.22\left(\mathrm{~s}, 2 \mathrm{H}, \mathrm{CH}_{2}-\mathrm{SO}_{2}\right), 4.14-4.08(\mathrm{q}, J=7.28 \mathrm{~Hz}, 1 \mathrm{H}, \mathrm{CH}-$ $\left.\mathrm{CH}_{3}\right), 1.60-1.58\left(\mathrm{~d}, J=7.28 \mathrm{~Hz}, 3 \mathrm{H}, \mathrm{CH}_{3}-\mathrm{CH}\right) \mathrm{ppm} ;{ }^{13} \mathrm{C}$ NMR (Dioxane, $100 \mathrm{MHz}) \delta$ : $176.8(\mathrm{CO}), 131.0,130.6$ (2CH aromatic), 129.3 (2CH aromatic), 129.1, 60.4, 52.1, $19.6 \mathrm{ppm}$; IR (KBr) v: 3424 ( $\mathrm{OH}$ of acid), 2974 (CH aromatic), 2822 ( $\mathrm{CH}$ aliphatic), $1751(\mathrm{C}=\mathrm{O}$ of $\mathrm{COOH}), 1599$ $(\mathrm{C}=\mathrm{C}), 1213,1169\left(\mathrm{SO}_{2}\right.$ two bands), $698(\mathrm{Ar}-\mathrm{H}) \mathrm{cm}^{-1} ; \mathrm{MS}$ : in $\mathrm{m} / \mathrm{z}$ [rel. \%]: 212.1 [22\%], 180.1 [81.5\%], 179.1 [91\%], 178.1 [85\%], 165.1 [M - $\left.\mathrm{PhCH}_{3}, 55 \%\right], 122.0$ [80\%], 121.0 [100\%], 77.0 [ $\left.\mathrm{Ph}^{+}, 71.4 \%\right], 64.0\left[\mathrm{SO}_{2}{ }^{+}, 54.6 \%\right], 51.0[28 \%]$. Anal calcd for $\mathrm{C}_{10} \mathrm{H}_{13} \mathrm{NO}_{4} \mathrm{~S}$ (243.28): C 49.37, H 5.39, N 5.76; found: C 49.29, H 5.28, N 5.94.

3-Mercapto-2-(phenylmethylsulfonamido)propanoic acid (1e): Yield $1.22 \mathrm{~g}(89 \%) ; \mathrm{mp} 171-173{ }^{\circ} \mathrm{C} ; \mathrm{R}_{\mathrm{f}}=0.49 ;{ }^{1} \mathrm{H}$ NMR $\left(\mathrm{D}_{2} \mathrm{O}, 400 \mathrm{MHz}\right) \delta: 7.49$ (s, 5H, Ar-H), 4.50-4.47 (dd, $\left.J_{1}=4.24 \mathrm{~Hz}, J_{2}=7.92 \mathrm{~Hz}, 1 \mathrm{H}, \mathrm{CH}_{2}-\mathrm{CH}-\mathrm{COOH}\right), 4.24(\mathrm{~s}$, $\left.2 \mathrm{H}, \mathrm{CH}_{2}-\mathrm{SO}_{2}\right), 3.55-3.50\left(\mathrm{dd}, J_{1}=4.24 \mathrm{~Hz}, J_{2}=20 \mathrm{~Hz}, 1 \mathrm{H}\right.$, $\mathrm{CHa}$ of $\mathrm{CH}_{2}-\mathrm{CH}$ ), 3.39-3.33 (dd, $J_{1}=7.92 \mathrm{~Hz}, J_{2}=20 \mathrm{~Hz}$, $1 \mathrm{H}, \mathrm{CHb}$ of $\mathrm{CH}_{2}-\mathrm{CH}$ ) ppm; ${ }^{13} \mathrm{C}$ NMR (Dioxane, $100 \mathrm{MHz}$ ) $\delta$ : $171.5(\mathrm{CO}), 132.6,131.3(2 \mathrm{CH}$ aromatic), $129.5(2 \mathrm{CH}$ aromatic), 128.7, 57.8, 52.7, $37.2 \mathrm{ppm}$; IR (KBr) v: 3439 (OH of acid), 2978 ( $\mathrm{CH}$ aromatic), 2832 ( $\mathrm{CH}$ aliphatic), 
$1728(\mathrm{C}=\mathrm{O}$ of $\mathrm{COOH}), 1618(\mathrm{C}=\mathrm{C}), 1207,1159\left(\mathrm{SO}_{2}\right.$ two bands), 810 (Ar-H) cm ${ }^{-1}$; MS: in m/z [rel. \%]: 214.1 [31.7\%], $123.0[100 \%], 122.0$ [90\%], $92.1\left[\mathrm{PhCH}_{3}{ }^{+}, 33 \%\right], 91.0$ $\left[\mathrm{PhCH}_{2}{ }^{+}, 88 \%\right.$ ], $77.0\left[\mathrm{Ph}^{+}, 8 \%\right], 65.0\left[\mathrm{HSO}_{2}{ }^{+}, 34 \%\right], 45.0$ [ $\left.{ }^{+} \mathrm{COOH}, 28 \%\right], 36.0$ [34\%]. Anal calcd for $\mathrm{C}_{10} \mathrm{H}_{13} \mathrm{NO}_{4} \mathrm{~S}_{2}$ (275.35): C 43.62, H 4.76, N 5.09; found: C 43.45, H 4.94, N 5.07 .

4-(Methylthio)-2-(phenylmethylsulfonamido)butanoic acid (1f): Yield $1.33 \mathrm{~g} \mathrm{(87.7 \% );} \mathrm{mp} \mathrm{89-90}{ }^{\circ} \mathrm{C} ; \mathrm{R}_{\mathrm{f}}=0.68 ;{ }^{1} \mathrm{H}$ NMR $\left(\mathrm{D}_{2} \mathrm{O}, 400 \mathrm{MHz}\right) \delta: 7.47(\mathrm{~s}, 5 \mathrm{H}, \mathrm{Ar}-\mathrm{H}), 4.23(\mathrm{~s}, 2 \mathrm{H}$, $\mathrm{CH}_{2}-\mathrm{SO}_{2}$ ), 4.01-3.97 (t, $\left.J=8.84 \mathrm{~Hz}, 1 \mathrm{H}, \underline{\mathrm{CH}}-\mathrm{CH}_{2}\right), 2.76-$ 2.72 (t, $\left.J=7.40 \mathrm{~Hz}, 2 \mathrm{H}, \mathrm{CH}_{2}-\mathrm{CH}_{2}-\mathrm{S}\right), 2.33-2.29(\mathrm{~m}, 1 \mathrm{H}$, $\mathrm{CH}), 2.28-2.21(\mathrm{~m}, 1 \mathrm{H}, \mathrm{CH}), 2.19\left(\mathrm{~s}, 3 \mathrm{H}, \mathrm{CH}_{3}-\mathrm{S}\right) \mathrm{ppm} ;{ }^{13} \mathrm{C}$ NMR (Dioxane, $100 \mathrm{MHz}$ ) $\delta$ : 172.9 (CO), 132.6, 131.2 (2CH aromatic), 129.5 (2CH aromatic), 128.7, 57.9, 52.8, 29.8, 29.4, 14.7 ppm; IR (KBr) v: 3442 (OH of acid), 2974 (CH aromatic), 2833 ( $\mathrm{CH}$ aliphatic), 2774 ( $\mathrm{CH}$ aliphatic), $1742(\mathrm{C}=\mathrm{O}$ of $\mathrm{COOH}), 1590(\mathrm{C}=\mathrm{C}), 1211,1161\left(\mathrm{SO}_{2}\right.$ two bands), 698 (Ar-H) cm $\mathrm{cm}^{-1}$. Anal calcd for $\mathrm{C}_{12} \mathrm{H}_{17} \mathrm{NO}_{4} \mathrm{~S}_{2}$ (303.40): C 47.51, H 5.65, N 4.62; found: C 47.49, H 5.64, N 4.66 .

3-Methyl-2-(phenylmethylsulfonamido)butanoic acid (1g): Yield $1.34 \mathrm{~g}(98.7 \%) ; \mathrm{mp} 137-138{ }^{\circ} \mathrm{C} ; \mathrm{R}_{\mathrm{f}}=0.83 ;{ }^{1} \mathrm{H}$ NMR $\left(\mathrm{D}_{2} \mathrm{O}, 400 \mathrm{MHz}\right) \delta: 7.49(\mathrm{~s}, 5 \mathrm{H}, \mathrm{Ar}-\mathrm{H}), 4.25(\mathrm{~s}, 2 \mathrm{H}$, $\left.\mathrm{CH}_{2}-\mathrm{SO}_{2}\right), 4.04-4.03(\mathrm{~d}, J=4.4 \mathrm{~Hz}, 1 \mathrm{H}, \mathrm{CH}-\mathrm{CH}-\mathrm{COOH})$, 2.47-2.38 (m, 1H, CH-CH- $\left.\left(\mathrm{CH}_{3}\right)_{2}\right), 1.15-1.13(\mathrm{~d}, J=7.04$ $\left.\mathrm{Hz}, 3 \mathrm{H}, \mathrm{CH}_{3}-\mathrm{CH}\right), 1.13-1.11\left(\mathrm{~d}, J=7.08 \mathrm{~Hz}, 3 \mathrm{H}, \mathrm{CH}_{3}-\mathrm{CH}\right)$ ppm; ${ }^{13} \mathrm{C}$ NMR (Dioxane, $\left.100 \mathrm{MHz}\right) \delta: 172.4$ (CO), 132.5 , 131.2 (2CH aromatic), 129.5 (2CH aromatic), 128.9, 59.2, 57.7, 29.8, 18.1, $17.8 \mathrm{ppm}$; IR ( $\mathrm{KBr}) v: 3447$ (OH of acid), 2974 (CH aromatic), 2833 (CH aliphatic), 2783 (CH aliphatic), $1730(\mathrm{C}=\mathrm{O}$ of $\mathrm{COOH}), 1618(\mathrm{C}=\mathrm{C}), 1225,1165$ ( $\mathrm{SO}_{2}$ two bands), $700(\mathrm{Ar}-\mathrm{H}) \mathrm{cm}^{-1}$; MS: in $\mathrm{m} / \mathrm{z}$ [rel. \%]: $271.1\left[\mathrm{M}^{+}, 14 \%\right], 91.0\left[\mathrm{PhCH}_{2}{ }^{+}, 30 \%\right], 75.0$ [65\%], 72.1 [100\%], 55.0 [79\%], 29.0 [50\%]. Anal calcd for $\mathrm{C}_{12} \mathrm{H}_{17} \mathrm{NO}_{4} \mathrm{~S}$ (271.34): C 53.12, H 6.32, N 5.16; found: C 53.31, H 6.50, N 5.20 .

3-Hydroxy-2-(phenylmethylsulfonamido)butanoic

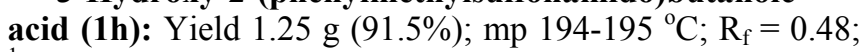
${ }^{1} \mathrm{H}$ NMR $\left(\mathrm{D}_{2} \mathrm{O}, 400 \mathrm{MHz}\right) \delta$ : 7.49 (s, 5H, Ar-H), 4.48-4.46 (m, $1 \mathrm{H}, \mathrm{CH}), 4.24\left(\mathrm{~s}, 2 \mathrm{H}, \mathrm{CH}_{2}-\mathrm{SO}_{2}\right), 4.05-4.04(\mathrm{~d}, J=4.4$ $\mathrm{Hz}, 1 \mathrm{H}, \mathrm{CH}-\mathrm{CH}-\mathrm{COOH}), 1.42-1.41(\mathrm{~d}, J=6.64 \mathrm{~Hz}, 3 \mathrm{H}$, $\mathrm{CH}_{3}-\mathrm{CH}$ ) ppm; ${ }^{13} \mathrm{C}$ NMR (Dioxane, $\left.100 \mathrm{MHz}\right) \delta: 171.5$ (CO), 132.5, 131.2 (2CH aromatic), 129.5 (2CH aromatic), 128.9, 66.1, 59.4, 57.7, 19.8 ppm; IR (KBr) v: $3404(\mathrm{OH}$ of acid), 2976 ( $\mathrm{CH}$ aromatic), 2824 ( $\mathrm{CH}$ aliphatic), $1740(\mathrm{C}=\mathrm{O}$ of $\mathrm{COOH}), 1601(\mathrm{C}=\mathrm{C}), 1219,1157\left(\mathrm{SO}_{2}\right.$ two bands $), 700$ (Ar-H) $\mathrm{cm}^{-1}$. Anal calcd for $\mathrm{C}_{11} \mathrm{H}_{15} \mathrm{NO}_{5} \mathrm{~S}$ (273.31): $\mathrm{C} 48.34$, H 5.53, N 5.12; found: C 48.29, H 5.61, N 4.98.

5-Amino-5-oxo-2-(phenylmethylsulfonamido) pentanoic acid (1i): Yield $1.41 \mathrm{~g}(94.1 \%) ; \mathrm{mp} 211-214^{\circ} \mathrm{C} ; \mathrm{R}_{\mathrm{f}}=$ 0.38 ; ${ }^{1} \mathrm{H}$ NMR $\left(\mathrm{D}_{2} \mathrm{O}, 400 \mathrm{MHz}\right) \delta: 7.48$ (s, 5H, Ar-H), 4.47$4.43\left(\mathrm{dd}, J_{1}=5.04 \mathrm{~Hz}, J_{2}=14.32 \mathrm{~Hz}, 1 \mathrm{H}, \mathrm{HOOC}-\mathrm{CH}-\mathrm{CH}_{2}\right)$, $4.23\left(\mathrm{~s}, 2 \mathrm{H}, \mathrm{CH}_{2}-\mathrm{SO}_{2}\right), 2.62-2.55(\mathrm{~m}, 1 \mathrm{H}, \mathrm{CH}), 2.49-2.44$ $\left(\mathrm{dd}, J_{1}=9.2 \mathrm{~Hz}, J_{2}=18.72 \mathrm{~Hz}, 2 \mathrm{H}, \mathrm{CH}_{2}-\mathrm{CH}_{2}-\mathrm{CON}\right.$ ), $2.27-$ $2.20(\mathrm{~m}, 1 \mathrm{H}, \mathrm{CH}) \mathrm{ppm} ;{ }^{13} \mathrm{C}$ NMR (Dioxane, $\left.100 \mathrm{MHz}\right) \delta$ : 181.1 (CO of acid), 163.2 (CO of amide), 132.5, 131.2 (2CH aromatic), 129.5 (2CH aromatic), 128.9, 59.2, 57.7, 30.5, $26.2 \mathrm{ppm}$; IR (KBr) v: 3246 (OH of acid), 3075, $3053(\mathrm{NH}$ two bands), 2983 ( $\mathrm{CH}$ aromatic), 2951 (CH aliphatic), 1703
$(\mathrm{C}=\mathrm{O}$ of $\mathrm{COOH}), 1659(\mathrm{C}=\mathrm{O}$ amide $), 1412(\mathrm{OH}$ bending inplane), 1221, 1193 ( $\mathrm{SO}_{2}$ two bands), $696(\mathrm{Ar}-\mathrm{H}), 631(\mathrm{~N}-\mathrm{H}$ bending with wagging) $\mathrm{cm}^{-1}$. Anal calcd for $\mathrm{C}_{12} \mathrm{H}_{16} \mathrm{~N}_{2} \mathrm{O}_{5} \mathrm{~S}$ (300.34): C 47.99, H 5.37, N 9.33; found: C 48.03, H 5.56, N 9.41 .

3-Phenyl-2-(phenylmethylsulfonamido)propanoic acid (1j): Yield $=1.58 \mathrm{~g}(98.8 \%) ; \mathrm{mp} 158-159^{\circ} \mathrm{C}(\mathrm{dec}) ; \mathrm{R}_{\mathrm{f}}=$ 0.84; ${ }^{1} \mathrm{H}$ NMR $\left(\mathrm{D}_{2} \mathrm{O}, 400 \mathrm{MHz}\right) \delta: 7.46(\mathrm{~s}, 10 \mathrm{H}, 2 \times \mathrm{Ar}-\mathrm{H})$, $4.34-4.31\left(\mathrm{dd}, J_{1}=5.60 \mathrm{~Hz}, J_{2}=13.28 \mathrm{~Hz}, 1 \mathrm{H}, \mathrm{PhCH}_{2}-\mathrm{CH}-\right.$ $\mathrm{COOH}), 4.22\left(\mathrm{~s}, 2 \mathrm{H}, \mathrm{CH}_{2}-\mathrm{SO}_{2}\right), 3.41-3.36\left(\mathrm{dd}, J_{1}=5.60 \mathrm{~Hz}\right.$, $J_{2}=20.00 \mathrm{~Hz}, \mathrm{CHa}$ of $\left.\mathrm{CH}_{2}-\mathrm{Ph}\right), 3.28-3.22\left(\mathrm{dd}, J_{1}=7.60 \mathrm{~Hz}\right.$, $J_{2}=20 \mathrm{~Hz}, 1 \mathrm{H}, \mathrm{CHb}$ of $\mathrm{CH}_{2}-\mathrm{Ph}$ ) ppm; ${ }^{13} \mathrm{C}$ NMR (Dioxane, $100 \mathrm{MHz}) \delta: 172.3$ (CO of acid), 134.8, 132.6, $131.2(2 \mathrm{CH}$ aromatic), 130.3 (2CH aromatic), 130.1 (2CH aromatic), 129.5 (2CH aromatic), 128.9 (2CH aromatic), 57.7, 55.0, 36.4 ppm; IR (KBr) v: 3441 (OH of acid), $2974(\mathrm{NH}), 2822$ ( $\mathrm{CH}$ aromatic), $2776(\mathrm{CH}$ aliphatic), $1740(\mathrm{C}=\mathrm{O}$ of $\mathrm{COOH})$, $1609(\mathrm{C}=\mathrm{C}), 1221,1171\left(\mathrm{SO}_{2}\right.$ two bands), $702(\mathrm{Ar}-\mathrm{H}), 623$ (N-H bending) $\mathrm{cm}^{-1}$; MS: in $\mathrm{m} / \mathrm{z}$ [rel. \%]: 270.1 [4\%], 212.1 [12\%], 180.1 [90\%], 179.1 [95\%], 178.1 [100\%], 165.1 [60\%], 122.0 [34\%], 121.0 [67\%], $64\left[\mathrm{SO}_{2}{ }^{+}, 70 \%\right]$. Anal calcd for $\mathrm{C}_{16} \mathrm{H}_{17} \mathrm{NO}_{4} \mathrm{~S}$ (319.38): C 60.17, H 5.37, N 4.39; found: C 59.98, H 5.21, N 4.42.

3-(4-(Benzylsulfonyloxy)phenyl)-2-(phenylmethylsulfonamido)propanoic acid (1k): Yield $=2.19 \mathrm{~g}(89.6 \%)$; mp 195-196 ${ }^{\circ} \mathrm{C} ; \mathrm{R}_{\mathrm{f}}=0.82 ;{ }^{1} \mathrm{H}$ NMR $\left(\mathrm{D}_{2} \mathrm{O}, 400 \mathrm{MHz}\right) \delta: 7.43$ (s, $10 \mathrm{H}, 2 \times \mathrm{Ar}-\mathrm{H}), 7.22-7.20(\mathrm{~d}, J=8.4 \mathrm{~Hz}, 2 \mathrm{H}, \mathrm{OTs}-\mathrm{H})$, 6.93-6.91 (d, $J=8.4 \mathrm{~Hz}, 2 \mathrm{H}$, OTs-H), 4.31-4.28 (dd, $J_{1}=$ $\left.5.60 \mathrm{~Hz}, J_{2}=13.08 \mathrm{~Hz}, 1 \mathrm{H}, \mathrm{PhCH}_{2}-\mathrm{CH}-\mathrm{COOH}\right), 4.19$ (s, 4H, $\left.2 \times \mathrm{CH}_{2}-\mathrm{SO}_{2}\right), 3.31-3.26\left(\mathrm{dd}, J_{1}=5.60 \mathrm{~Hz}, J_{2}=20.00 \mathrm{~Hz}\right.$, $\mathrm{CHa}$ of $\mathrm{CH}_{2}-\mathrm{Ph}$ ), 3.19-3.14 (dd, $J_{1}=7.52 \mathrm{~Hz}, J_{2}=20 \mathrm{~Hz}$, $1 \mathrm{H}, \mathrm{CHb}$ of $\mathrm{CH}_{2}-\mathrm{Ph}$ ) ppm; ${ }^{13} \mathrm{C}$ NMR (Dioxane, $\left.100 \mathrm{MHz}\right) \delta$ : 172.8 (CO of acid), 156.5, 140.9, 132.0, $131.8(2 \mathrm{CH}$ aromatic), 131.3 (2CH aromatic), 129.6 (2CH aromatic), 129.0 (2CH aromatic), 126.6, 123.6 (2CH aromatic), 117.1 (2CH aromatic), 111.8 (2CH aromatic), 58.4, 55.4, $36.1 \mathrm{ppm}$; IR $(\mathrm{KBr})$ v: $3435(\mathrm{OH}$ of acid), $3167(\mathrm{NH}), 3028(\mathrm{CH}$ aromatic), 2949 ( $\mathrm{CH}$ aliphatic), $1724(\mathrm{C}=\mathrm{O}$ of $\mathrm{COOH}), 1597$ $(\mathrm{C}=\mathrm{C}), 1194,1148$ ( $\mathrm{SO}_{2}$ two bands), 789 (S-OR ester), 694 (Ar-H), 631 (N-H bending) $\mathrm{cm}^{-1}$; MS: in $\mathrm{m} / \mathrm{z}$ [rel. \%]: 180.1 [74\%], 179.1 [90\%], 178.1 [100\%], 165.1 [30\%], 122.0 [10\%], $91.1\left[\mathrm{PhCH}_{2}^{+}, 47 \%\right], 64\left[\mathrm{SO}_{2}^{+}, 24 \%\right]$. Anal calcd for $\mathrm{C}_{23} \mathrm{H}_{23} \mathrm{NO}_{7} \mathrm{~S}_{2}$ (489.57): C 56.43, H 4.76, N 2.86; found: C $56.38, \mathrm{H} 4.79, \mathrm{~N} 2.69$.

Synthesis of $N, N$-diethyl substituted amide of benzenemethanesulfonamide derivatives (2). To a solution of appropriate arylmethylsulfonamides (1a-k) $(2.96 \mathrm{mmol})$ in $\mathrm{H}_{2} \mathrm{O}(10 \mathrm{~mL})$ in a streaming flow of nitrogen gas, was added oxalyl chloride ( $0.34 \mathrm{~mL}, 3.85 \mathrm{mmol}, 1.30$ eq.) via dropping pipette followed by carefully controlled addition of 1 drop of $N, N$-dimethylformamide (DMF). The resulting mixture was stirred at room temperature for $2 \mathrm{~h}$ to get crude acid chloride which was kept air-tighted prior to use. In a separate $250 \mathrm{ml}$ three-necked round bottom flask, equipped with a magnetic stirring bar, was added $\mathrm{Na}_{2} \mathrm{CO}_{3}(0.628 \mathrm{~g}, 5.92 \mathrm{mmol}, 2$ equiv.) to $\mathrm{H}_{2} \mathrm{O}(10 \mathrm{~mL})$ followed by diethyl amine (DEA) $(0.4 \mathrm{~mL}, 3.85 \mathrm{mmol}, 1.3$ equiv.) in continuous stirring and cooled to $-15{ }^{\circ} \mathrm{C}$. Then, earlier kept acid chloride was added in such a way to maintain the internal temperature of the reaction mixture at around $-10{ }^{\circ} \mathrm{C}$. The reacting mixture was 
then stirred at $-10{ }^{\circ} \mathrm{C}$ for $1 \mathrm{~h}$; at $0{ }^{\circ} \mathrm{C}$ for $1 \mathrm{~h}$ and finally at room temperature for $1 \mathrm{~h}$. The reaction was terminated, worked up by acidifying with $2 \mathrm{~N} \mathrm{HCl}$ and concentrated in rotary evaporator. The clear solution obtained was freezedried to get crude solid product which was purified by column chromatography $\left(\mathrm{CHCl}_{3} / \mathrm{CH}_{3} \mathrm{OH}, 3: 1\right)$ to afford $N, N$ diethylamide of benzenemethanesulfonamide derivatives (2a-k).

\section{1-(Benzylsulfonyl)- $N$, $N$-diethylpyrrolidine-2-}

carboxamide (2a): Yield $=0.94 \mathrm{~g}(97.9 \%)$; $\mathrm{mp} 185-187{ }^{\circ} \mathrm{C}$; $\mathrm{R}_{\mathrm{f}}=0.71 ;{ }^{1} \mathrm{H}$ NMR $\left(\mathrm{D}_{2} \mathrm{O}, 400 \mathrm{MHz}\right) \delta: 7.53(\mathrm{~s}, 5 \mathrm{H}, \mathrm{Ar}-\mathrm{H})$, 4.54-4.50 (m, 1H, CH-CON), $4.28\left(\mathrm{~s}, 2 \mathrm{H}, \mathrm{CH}_{2}-\mathrm{SO}_{2}\right), 3.56-$ $3.54\left(\mathrm{~m}, 2 \mathrm{H}, \mathrm{CH}_{2}-\mathrm{N}\right), 3.19-3.14(\mathrm{q}, J=7.28 \mathrm{~Hz}, 4 \mathrm{H}, 2 \times$ $\left.\mathrm{CH}_{2}-\mathrm{CH}_{3}\right), 2.54-2.51(\mathrm{~m}, 1 \mathrm{H}, \mathrm{CH}), 2.28-2.24(\mathrm{~m}, 1 \mathrm{H}, \mathrm{CH})$, 2.16-2.14 (m, 2H, $\left.\mathrm{CH}_{2}\right), 1.39-1.35(\mathrm{t}, J=7.28 \mathrm{~Hz}, 6 \mathrm{H}, 2 \times$ $\underline{\mathrm{CH}}_{3}-\mathrm{CH}_{2}$ ) ppm; ${ }^{13} \mathrm{C}$ NMR (Dioxane, $100 \mathrm{MHz}$ ) $\delta: 173.2$ $(\mathrm{C}=\mathrm{O}), 132.6,131.1$ (2CH aromatic), 129.4 (2CH aromatic), 128.8, 60.8, 57.7, 47.1, $43.3\left(2 \mathrm{CH}_{2}\right), 29.2,24.2,11.4\left(2 \mathrm{CH}_{3}\right)$ ppm. Anal calcd for $\mathrm{C}_{16} \mathrm{H}_{24} \mathrm{~N}_{2} \mathrm{O}_{3} \mathrm{~S}$ (324.45): C 59.23, $\mathrm{H}$ 7.46, N 8.63, found: C 59.09, H 7.46, N 8.48.

1-(Benzylsulfonyl)- $\boldsymbol{N}, \boldsymbol{N}$-diethylpiperidine-2-carboxamide (2b): Yield $=0.99 \mathrm{~g}(99.0 \%) ; \mathrm{mp} 210-211{ }^{\circ} \mathrm{C} ; \mathrm{R}_{\mathrm{f}}=$ $0.72 ;{ }^{1} \mathrm{H}$ NMR $\left(\mathrm{D}_{2} \mathrm{O}, 400 \mathrm{MHz}\right) \delta: 7.54$ (s, 5H, Ar-H), 4.30 (s, $\left.2 \mathrm{H}, \underline{\mathrm{CH}}_{2}-\mathrm{SO}_{2}\right), 4.08-4.04\left(\mathrm{dd}, J_{1}=3.52 \mathrm{~Hz}, J_{2}=20 \mathrm{~Hz}\right.$, $1 \mathrm{H}, \mathrm{CH}$ of $\left.\mathrm{CH}_{2}-\mathrm{N}\right), 3.60-3.57$ (m, 1H, $\left.\mathrm{CH}-\mathrm{CON}\right), 3.21-3.16$ $\left(\mathrm{q}, J=7.20 \mathrm{~Hz}, 4 \mathrm{H}, 2 \times \underline{\mathrm{CH}_{2}}-\mathrm{CH}_{3}\right), 2.42-2.38\left(\mathrm{dd}, J_{1}=3.32\right.$ $\mathrm{Hz}, J_{2}=20 \mathrm{~Hz}, 1 \mathrm{H}, \mathrm{CH}$ of $\left.\mathrm{CH}_{2}-\mathrm{N}\right), 2.03-1.99\left(\mathrm{~m}, 2 \mathrm{H}, \mathrm{CH}_{2}\right)$, 1.86-1.73 (m, 3H, CH \& $\left.\mathrm{CH}_{2}\right), 1.40-1.37(\mathrm{t}, J=7.20 \mathrm{~Hz}, 6 \mathrm{H}$, $2 \times \underline{\mathrm{CH}}_{3}-\mathrm{CH}_{2}$ ) ppm; ${ }^{13} \mathrm{C}$ NMR (Dioxane, $100 \mathrm{MHz}$ ) $\delta: 173.5$ $(\mathrm{C}=\mathrm{O}), 132.9,131.1$ (2CH aromatic), 129.8 (2CH aromatic), 128.6, 60.6, 57.8, 47.4, $43.4\left(\mathrm{CH}_{2}\right), 29.4,24.2,18.1,11.4$ $\left(\mathrm{CH}_{3}\right)$ ppm; IR (KBr) v: $3028(\mathrm{CH}$ aromatic), 2951 (CH aliphatic), $1720(\mathrm{C}=\mathrm{O}), 1593(\mathrm{C}=\mathrm{C}), 1188,1148\left(\mathrm{SO}_{2}\right.$ two bands), $696(\mathrm{Ar}-\mathrm{H}) \mathrm{cm}^{-1}$; anal calcd for $338.47 \mathrm{C}_{17} \mathrm{H}_{26} \mathrm{~N}_{2} \mathrm{O}_{3} \mathrm{~S}$ : C, 60.33; H, 7.74; N, 8.28; found: C, 60.29; H, 7.94; N, 7.98.

$\mathbf{N}, \boldsymbol{N}$-Diethyl-2-(phenylmethylsulfonamido)acetamide (2c): Yield $=0.78 \mathrm{~g}(92.6 \%) ; \mathrm{mp} 213-215{ }^{\circ} \mathrm{C} ; \mathrm{R}_{\mathrm{f}}=0.51 ;{ }^{1} \mathrm{H}$ NMR $\left(\mathrm{D}_{2} \mathrm{O}, 400 \mathrm{MHz}\right) \delta: 7.53(\mathrm{~s}, 5 \mathrm{H}, \mathrm{Ar}-\mathrm{H}), 4.29(\mathrm{~s}, 2 \mathrm{H}$, $\mathrm{CH}_{2}-\mathrm{SO}_{2}$ ), 4.01 (s, 2H, $\mathrm{CH}_{2}-\mathrm{CON}$ ), 3.20-3.14 (q, $J=7.30$ $\left.\mathrm{Hz}, 4 \mathrm{H}, 2 \times \underline{\mathrm{CH}}_{2}-\mathrm{CH}_{3}\right), 1.39-1.36(\mathrm{t}, J=7.30 \mathrm{~Hz}, 6 \mathrm{H}, 2 \times$ $\underline{\mathrm{CH}}_{3}-\mathrm{CH}_{2}$ ) ppm; ${ }^{13} \mathrm{C}$ NMR (Dioxane, $100 \mathrm{MHz}$ ) $\delta: 174.1$ $(\mathrm{C}=\mathrm{O}), 133.4,131.6$ (2CH aromatic), 129.5 (2CH aromatic), 128.6, 57.7, 49.8, $43.1\left(\mathrm{CH}_{2}\right), 11.4\left(\mathrm{CH}_{3}\right)$ ppm; IR $(\mathrm{KBr})$ v: $3217(\mathrm{~N}-\mathrm{H}), 3036$ (CH aromatic), 2947 (CH aliphatic), 1712 $(\mathrm{C}=\mathrm{O}), 1601(\mathrm{C}=\mathrm{C}), 1219,1194,\left(\mathrm{SO}_{2}\right.$ two bands), 694 (ArH) $\mathrm{cm}^{-1}$. Anal calcd for $\mathrm{C}_{13} \mathrm{H}_{20} \mathrm{~N}_{2} \mathrm{O}_{3} \mathrm{~S}$ (284.38): $\mathrm{C} 54.91, \mathrm{H}$ 7.09, N 9.85; found: C 55.13, H 6.94, N 10.08.

$\boldsymbol{N}, \boldsymbol{N}$-Diethyl-2-(phenylmethylsulfonamido)propanemide (2d): Yield $=0.87 \mathrm{~g}(98.5 \%) ; \mathrm{mp} 238-240{ }^{\circ} \mathrm{C} ; \mathrm{R}_{\mathrm{f}}=$ 0.56; ${ }^{1} \mathrm{H}$ NMR $\left(\mathrm{D}_{2} \mathrm{O}, 400 \mathrm{MHz}\right) \delta: 7.50$ (s, 5H, Ar-H), 4.26 (s, $2 \mathrm{H}, \underline{\mathrm{CH}}_{2}-\mathrm{SO}_{2}$ ), $4.22-4.16$ (q, $\left.J=7.28 \mathrm{~Hz}, 1 \mathrm{H}, \underline{\mathrm{CH}}-\mathrm{CH}_{3}\right)$, 3.18-3.12 (q, $\left.J=7.32 \mathrm{~Hz}, 4 \mathrm{H}, 2 \times \mathrm{CH}_{2}-\mathrm{CH}_{3}\right), 1.65-1.63(\mathrm{~d}, J$ $\left.=7.28 \mathrm{~Hz}, 3 \mathrm{H}, \underline{\mathrm{CH}}_{3}-\mathrm{CH}\right), 1.37-1.33(\mathrm{t}, J=7.32 \mathrm{~Hz}, 6 \mathrm{H}, 2 \times$ $\underline{\mathrm{CH}}_{3}-\mathrm{CH}_{2}$ ) ppm; ${ }^{13} \mathrm{C}$ NMR (Dioxane, $100 \mathrm{MHz}$ ) $\delta: 173.8$ $(\mathrm{C}=\mathrm{O}), 132.7,131.2$ (2CH aromatic), 129.5 (2CH aromatic), 128.9, 57.7, 49.8, 43.1 $\left(\mathrm{CH}_{2}\right), 16.2,11.4\left(\mathrm{CH}_{3}\right) \mathrm{ppm}$; IR $(\mathrm{KBr})$ v: $3058(\mathrm{~N}-\mathrm{H}), 3036(\mathrm{CH}$ aromatic), $2951(\mathrm{CH}$ aliphatic), $1719(\mathrm{C}=\mathrm{O}), 1601(\mathrm{C}=\mathrm{C}), 1219,1196,1148\left(\mathrm{SO}_{2}\right.$ two bands), $696(\mathrm{Ar}-\mathrm{H}) \mathrm{cm}^{-1}$. Anal calcd for $\mathrm{C}_{14} \mathrm{H}_{22} \mathrm{~N}_{2} \mathrm{O}_{3} \mathrm{~S}$
(298.41): C 56.35, H 7.43, N 9.39; found: C 56.11, H 7.33, N 9.28 .

$N, N$-Diethyl-3-mercapto-2(phenylmethylsulfonamido)propanamide (2e): Yield 0.87 g (89.0\%); mp 198-200 ${ }^{\circ} \mathrm{C} ; \mathrm{R}_{\mathrm{f}}=0.71 ;{ }^{1} \mathrm{H}$ NMR $\left(\mathrm{D}_{2} \mathrm{O}, 400\right.$ MHz) $\delta: 7.46$ (s, 5H, Ar-H), 4.54-4.51 (dd, $J_{1}=4.24 \mathrm{~Hz}, J_{2}=$ $\left.7.92 \mathrm{~Hz}, 1 \mathrm{H}, \mathrm{CH}_{2}-\mathrm{CH}-\mathrm{COOH}\right), 4.21$ (s, 2H, $\mathrm{CH}_{2}-\mathrm{SO}_{2}$ ), 3.57$3.52\left(\mathrm{dd}, J_{1}=4.24 \mathrm{~Hz}, J_{2}=20 \mathrm{~Hz}, 1 \mathrm{H}, \mathrm{CHa}\right.$ of $\left.\mathrm{CH}_{2}-\mathrm{CH}\right)$, $3.41-3.36\left(\mathrm{dd}, J_{1}=7.92 \mathrm{~Hz}, J_{2}=20 \mathrm{~Hz}, 1 \mathrm{H}, \mathrm{CHb}\right.$ of $\mathrm{CH}_{2-}$ $\mathrm{CH}$ ), 3.18-3.12 (q, $\left.J=7.35 \mathrm{~Hz}, 4 \mathrm{H}, 2 \times \mathrm{CH}_{2}-\mathrm{CH}_{3}\right), 1.37-1.33$ $\left(\mathrm{t}, J=7.35 \mathrm{~Hz}, 6 \mathrm{H}, 2 \times \mathrm{CH}_{3}-\mathrm{CH}_{2}\right.$ ) ppm; ${ }^{13} \mathrm{C}$ NMR (Dioxane, $100 \mathrm{MHz}) \delta: 173.5(\mathrm{C}=\mathrm{O}), 132.7,131.2$ (2CH aromatic), 129.5 (2CH aromatic), 128.9, 57.4, 49.5, $43.1\left(\mathrm{CH}_{2}\right), 35.5$, $11.4\left(\mathrm{CH}_{3}\right)$ ppm; IR (KBr) v: 2997 (CH aromatic), $2911(\mathrm{CH}$ aliphatic), $1719(\mathrm{C}=\mathrm{O}), 1591(\mathrm{C}=\mathrm{C}), 1220,1194,1144\left(\mathrm{SO}_{2}\right.$ two bands), 696 (Ar-H) $\mathrm{cm}^{-1}$. Anal calcd for $\mathrm{C}_{14} \mathrm{H}_{22} \mathrm{~N}_{2} \mathrm{O}_{3} \mathrm{~S}_{2}$ (330.47): C 50.88, H 6.71, N 8.48; found: C 50.71, H 6.99, N 7.97.

$\boldsymbol{N}, \boldsymbol{N}$-Diethyl-4-(methylthio)-2-(phenylmethylsulfonamido) butanamide (2f): Yield $=0.96 \mathrm{~g}(90.6 \%)$; mp 170-172 ${ }^{\circ} \mathrm{C}$; $\mathrm{R}_{\mathrm{f}}=0.65 ;{ }^{1} \mathrm{H}$ NMR $\left(\mathrm{D}_{2} \mathrm{O}, 400 \mathrm{MHz}\right) \delta: 7.49(\mathrm{~s}, 5 \mathrm{H}, \mathrm{Ar}-\mathrm{H})$, $4.24\left(\mathrm{~s}, 2 \mathrm{H}, \underline{\mathrm{CH}}_{2}-\mathrm{SO}_{2}\right), 4.23-4.20(\mathrm{t}, \mathrm{J}=6.72 \mathrm{~Hz}, 1 \mathrm{H}, \underline{\mathrm{CH}}-$ $\left.\mathrm{CH}_{2}\right), 3.16-3.10\left(\mathrm{q}, J=7.32 \mathrm{~Hz}, 4 \mathrm{H}, 2 \times \mathrm{CH}_{2}-\mathrm{CH}_{3}\right), 2.77-$ $2.73\left(\mathrm{t}, J=7.40 \mathrm{~Hz}, 2 \mathrm{H}, \mathrm{S}-\mathrm{CH}_{2}-\mathrm{CH}_{2}\right), 2.34-2.24(\mathrm{~m}, 2 \mathrm{H}$, $\left.\mathrm{CH}-\mathrm{CH}_{2}-\mathrm{CH}_{2}-\mathrm{S}\right), 2.19$ (s, 3H, $\left.\mathrm{CH}_{3}-\mathrm{S}\right), 1.35-1.31$ (t, $J=7.32$ $\mathrm{Hz}, 6 \mathrm{H}, 2 \times \underline{\mathrm{CH}}_{3}-\mathrm{CH}_{2}$ ) ppm; ${ }^{13} \mathrm{C} \mathrm{NMR}$ (Dioxane, $100 \mathrm{MHz}$ ) $\delta: 173.8,132.7,131.2(2 \mathrm{CH}$ aromatic), $129.5,(2 \mathrm{CH}$ aromatic), 57.7, 49.8, $43.1\left(\mathrm{CH}_{2}\right), 30.8,29.5,16.2,11.4\left(\mathrm{CH}_{3}\right)$ ppm; IR (KBr) v: 3028 ( $\mathrm{CH}$ aromatic), 2945 ( $\mathrm{CH}$ aliphatic), $1722(\mathrm{C}=\mathrm{O}), 1620(\mathrm{C}=\mathrm{C}), 1200,1126\left(\mathrm{SO}_{2}\right.$ two bands) 698 $(\mathrm{Ar}-\mathrm{H}) \mathrm{cm}^{-1}$; anal calcd for $358.53 \mathrm{C}_{16} \mathrm{H}_{26} \mathrm{~N}_{2} \mathrm{O}_{3} \mathrm{~S}_{2}$ : C, 53.60; H, 7.31; N, 7.81; found: C, 53.55; H, 7.22; N, 7.69.

$\mathrm{N}, \mathrm{N}$-Diethyl-3-methyl-2-(phenylmethylsulfonamido)butanemide (2g): Yield $=0.94 \mathrm{~g}(97.3 \%)$; mp $226-230{ }^{\circ} \mathrm{C}$; $\mathrm{R}_{\mathrm{f}}=0.69 ;{ }^{1} \mathrm{H}$ NMR $\left(\mathrm{D}_{2} \mathrm{O}, 400 \mathrm{MHz}\right) \delta: 7.50(\mathrm{~s}, 5 \mathrm{H}, \mathrm{Ar}-\mathrm{H})$, 4.25 (s, $2 \mathrm{H}, \underline{\mathrm{CH}}_{2}-\mathrm{SO}_{2}$ ), 4.00-3.97 (d, J = 2.84 Hz, 1H, CHCH-CON), 3.17-3.11 (q, $\left.J=7.32 \mathrm{~Hz}, 4 \mathrm{H}, 2 \times \underline{\mathrm{CH}_{2}}-\mathrm{CH}_{3}\right)$, 2.45-2.38 (m, 1H, CH), 1.36-1.32 (t, $J=7.32 \mathrm{~Hz}, 6 \mathrm{H}, 2 \times$ $\left.\mathrm{CH}_{3}-\mathrm{CH}_{2}\right), 1.15-1.13\left(\mathrm{~d}, J=7.00 \mathrm{~Hz}, 3 \mathrm{H}, \mathrm{CH}_{3}-\mathrm{CH}\right), 1.12-$ $1.10\left(\mathrm{~d}, J=7.00 \mathrm{~Hz}, 3 \mathrm{H}, \underline{\mathrm{CH}}_{3}-\mathrm{CH}\right) \mathrm{ppm} ;{ }^{13} \mathrm{C}$ NMR (Dioxane, $100 \mathrm{MHz}) \delta$ : $173.8(\mathrm{C}=\mathrm{O}), 132.7,131.2(2 \mathrm{CH}$ aromatic), 129.5 (2CH aromatic), 128.9, 60.8, 57.7, 49.8, 43.1 $\left(\mathrm{CH}_{2}\right), 16.2,15.5,11.4\left(\mathrm{CH}_{3}\right)$ ppm; IR $(\mathrm{KBr})$ v: $3053(\mathrm{CH}$ aromatic), 2945 (CH aliphatic), $1718(\mathrm{C}=\mathrm{O}), 1611(\mathrm{C}=\mathrm{C})$, 1219, 1194 ( $\mathrm{SO}_{2}$ two bands), $696(\mathrm{Ar}-\mathrm{H}) \mathrm{cm}^{-1}$. Anal calcd for $\mathrm{C}_{16} \mathrm{H}_{26} \mathrm{~N}_{2} \mathrm{O}_{3} \mathrm{~S}$ (326.46): $\mathrm{C}$ 58.87, $\mathrm{H} \mathrm{8.03,} \mathrm{N} 8.58$; found: $\mathrm{C}$ 59.01, H 7.96, N 8.61.

$\mathrm{N}, \mathrm{N}$-Diethyl-3-hydroxy-2-(phenylmethylsulfonamido) butanamide (2h): Yield $=0.78 \mathrm{~g}(80.2 \%)$; $\mathrm{mp} 245^{\circ} \mathrm{C}(\mathrm{dec})$; $\mathrm{R}_{\mathrm{f}}=0.53 ;{ }^{1} \mathrm{H}$ NMR $\left(\mathrm{D}_{2} \mathrm{O}, 400 \mathrm{MHz}\right) \delta: 7.55(\mathrm{~s}, 5 \mathrm{H}, \mathrm{Ar}-\mathrm{H})$, 4.54-4.52 (m, 1H, CH- $\left.\underline{\mathrm{CH}}-\mathrm{CH}_{3}\right), 4.31\left(\mathrm{~s}, 2 \mathrm{H}, \underline{\mathrm{CH}_{2}}-\mathrm{SO}_{2}\right)$, 4.09-4.08 (d, J = 3.96 Hz, 1H, CH-CH-CON), 3.22-3.17 (q, $J$ $\left.=7.32 \mathrm{~Hz}, 4 \mathrm{H}, 2 \times \underline{\mathrm{CH}_{2}}-\mathrm{CH}_{3}\right), 2.85(\mathrm{~s}, 1 \mathrm{H}, \mathrm{OH}), 1.49-1.47$ (d, $\left.J=6.60 \mathrm{~Hz}, 3 \mathrm{H}, \mathrm{CH}_{3}-\mathrm{CH}\right), 1.41-1.37$ (t, $J=7.32 \mathrm{~Hz}, 6 \mathrm{H}$, $2 \times \underline{\mathrm{CH}}_{3}-\mathrm{CH}_{2}$ ) ppm; ${ }^{13} \mathrm{C}$ NMR (Dioxane, $\left.100 \mathrm{MHz}\right) \delta: 173.5$ $(\mathrm{C}=\mathrm{O}), 132.9,131.3$ (2CH aromatic), 129.5 (2CH aromatic), 128.9, 61.2, 58.1, 49.5, $42.5\left(\mathrm{CH}_{2}\right), 16.6,11.1\left(\mathrm{CH}_{3}\right) \mathrm{ppm}$; IR $(\mathrm{KBr})$ v: $3396(\mathrm{OH}), 3030(\mathrm{CH}$ aromatic), $2945(\mathrm{CH}$ aliphatic), $1720(\mathrm{C}=\mathrm{O}), 1601(\mathrm{C}=\mathrm{C}), 1221,1194\left(\mathrm{SO}_{2}\right.$ two bands), 694 (Ar-H) cm $\mathrm{cm}^{-1}$. Anal calcd for $\mathrm{C}_{15} \mathrm{H}_{24} \mathrm{~N}_{2} \mathrm{O}_{4} \mathrm{~S}$ 
(328.43): C 54.86, H 7.37, N 8.53; found: C 54.71, H 7.26, N 8.65 .

$N^{1}, N^{1}$-Diethyl-2-(phenylmethylsulfonamido)pentanediamide (2i): Yield $=0.98 \mathrm{~g}(93.2 \%) ; \mathrm{mp} 251-253{ }^{\circ} \mathrm{C}$; $\mathrm{R}_{\mathrm{f}}=$ $0.58 ;{ }^{1} \mathrm{H}$ NMR $\left(\mathrm{D}_{2} \mathrm{O}, 400 \mathrm{MHz}\right) \delta: 7.51(\mathrm{~s}, 5 \mathrm{H}, \mathrm{Ar}-\mathrm{H}), 4.52-$ $4.48\left(\mathrm{dd}, J_{1}=5 \mathrm{~Hz}, J_{2}=14.32 \mathrm{~Hz}, 1 \mathrm{H}, \mathrm{NOC}-\mathrm{CH}-\mathrm{CH}_{2 a, b}\right)$, $4.26\left(\mathrm{~s}, 2 \mathrm{H}, \mathrm{CH}_{2}-\mathrm{SO}_{2}\right), 3.17-3.12(\mathrm{q}, J=7.32 \mathrm{~Hz}, 4 \mathrm{H}, 2 \times$ $\left.\mathrm{CH}_{2}-\mathrm{CH}_{3}\right), 2.67-2.60\left(\mathrm{~m}, 1 \mathrm{H}, \mathrm{CH}_{a}\right.$ of $\left.\mathrm{CH}_{2}\right), 2.53-2.48(\mathrm{t}, J=$ $\left.8 \mathrm{~Hz}, 2 \mathrm{H}, \mathrm{CO}-\mathrm{CH}_{2}-\mathrm{CH}_{2}\right), 2.30-2.24\left(\mathrm{~m}, 1 \mathrm{H}, \mathrm{CH}_{b}\right.$ of $\left.\mathrm{CH}_{2}\right)$, $1.37-1.33$ (t, $J=7.32 \mathrm{~Hz}, 6 \mathrm{H}, 2 \times \mathrm{CH}_{3}-\mathrm{CH}_{2}$ ) ppm; ${ }^{13} \mathrm{C} \mathrm{NMR}$ (Dioxane, $100 \mathrm{MHz}) \delta: 182.7(\mathrm{C}=\mathrm{O}), 177.1(\mathrm{C}=\mathrm{O}), 132.6$, 131.2 (2CH aromatic), 129.5 (2CH aromatic), 128.9, 57.7, 53.3, $43.1\left(\mathrm{CH}_{2}\right), 30.1,25.2,11.4\left(\mathrm{CH}_{3}\right) \mathrm{ppm}$; IR $(\mathrm{KBr}) \mathrm{v}$ : $3075(\mathrm{NH}), 3053(\mathrm{CH}$ aromatic), $2951(\mathrm{CH}$ aliphatic), 1703 $(\mathrm{C}=\mathrm{O}), 1659(\mathrm{C}=\mathrm{O}$ of $\mathrm{CON}), 1601(\mathrm{C}=\mathrm{C}), 1221,1193\left(\mathrm{SO}_{2}\right.$ two bands), $696(\mathrm{Ar}-\mathrm{H}) \mathrm{cm}^{-1}$. Anal calcd for $\mathrm{C}_{16} \mathrm{H}_{25} \mathrm{~N}_{3} \mathrm{O}_{4} \mathrm{~S}$ (355.46): C 54.06, H 7.09, N 11.82; found: C 53.95, H 6.88, $\mathrm{N} 12.01$.

$\mathrm{N}, \mathrm{N}$-Diethyl-3-phenyl-2-(phenylmethylsulfonamido) propanamide (2j): Yield $=1.00 \mathrm{~g}(90.2 \%) ; \mathrm{mp} 227-229^{\circ} \mathrm{C}$; $\mathrm{R}_{\mathrm{f}}=0.70 ;{ }^{1} \mathrm{H}$ NMR $\left(\mathrm{D}_{2} \mathrm{O}, 400 \mathrm{MHz}\right) \delta: 7.49(\mathrm{~s}, 10 \mathrm{H}, 2 \times \mathrm{Ar}-$ H), $4.39-4.36\left(\mathrm{dd}, J_{1}=5.60 \mathrm{~Hz}, J_{2}=7.60 \mathrm{~Hz}, 1 \mathrm{H}, \mathrm{NOC}-\underline{\mathrm{CH}}-\right.$ $\left.\mathrm{CH}_{2 a, b}\right), 4.24\left(\mathrm{~s}, 2 \mathrm{H}, \mathrm{CH}_{2}-\mathrm{SO}_{2}\right), 3.44-3.39\left(\mathrm{dd}, J_{1}=5.60 \mathrm{~Hz}\right.$, $J_{2}=20.00 \mathrm{~Hz}, 1 \mathrm{H}, \mathrm{CH}_{a}$ of $\left.\mathrm{CH}_{2 a, b}\right), 3.31-3.26\left(\mathrm{dd}, J_{1}=7.60\right.$ $\mathrm{Hz}, J_{2}=20.00 \mathrm{~Hz}, 1 \mathrm{H}, \mathrm{CH}_{b}$ of $\mathrm{CH}_{2 a, b}$ ), 3.16-3.10 (q, $J=7.32$ $\left.\mathrm{Hz}, 4 \mathrm{H}, 2 \times \mathrm{CH}_{2}-\mathrm{CH}_{3}\right), 2.78\left(\mathrm{~s}, 2 \mathrm{H}, \mathrm{CH}_{2}\right), 1.35-1.31(\mathrm{t}, J=$ $7.32 \mathrm{~Hz}, 6 \mathrm{H}, 2 \times \mathrm{CH}_{3}-\mathrm{CH}_{2}$ ) ppm; ${ }^{13} \mathrm{C}$ NMR (Dioxane, 100 MHz) $\delta: 172.4(\mathrm{C}=\mathrm{O}), 135.1,132.7,131.2(2 \mathrm{CH}$ aromatic), 130.3 (2CH aromatic), 130.1 (2CH aromatic), $129.5(2 \mathrm{CH}$ aromatic), 128.9 (2CH aromatic), 57.8, 55.2, $43.1\left(\mathrm{CH}_{2}\right)$, 36.5, $11.5\left(\mathrm{CH}_{3}\right)$ ppm; IR (KBr) v: $2976(\mathrm{NH}), 2828(\mathrm{CH}$ aromatic), $2774(\mathrm{CH}$ aliphatic $), 1736(\mathrm{C}=\mathrm{O}), 1620(\mathrm{C}=\mathrm{C})$, 1206, 1153, $1148\left(\mathrm{SO}_{2}\right.$ two bands), $698(\mathrm{Ar}-\mathrm{H}) \mathrm{cm}^{-1}$. Anal calcd for $\mathrm{C}_{20} \mathrm{H}_{26} \mathrm{~N}_{2} \mathrm{O}_{3} \mathrm{~S}$ (374.51): C 64.14, H 7.00, N 7.48; found: C 64.00, H 6.84, N 7.29.

\section{CONCLUSION}

In summary, out of all the sulfonamides, the one that involve benzenemethanesulfonamide framework has always been a great challenge to synthetic chemists in high yield. However, we have achieved the synthesis of this targeted benzenemethanesulfonamide derivatives (1a-k) in excellent yield in order to bridge the gap of its unmet demand. We have also succeeded in synthetic manipulation of these scaffolds to obtaining novel $N, N$-diethylalkanamide substituted sulfonamides (2a-k). However, the attempted effort on intramolecular cyclization of representative benzenemethanesulfonamide derivative (1a) and its $N, N$-diethylalkanamido substituted sulfonamide (2a) by silica mediated $\mathrm{AlCl}_{3}$ catalytic approach and direct ortho-metalation respectively, proved abortive as both conditions failed to deliver the expected benzothiazepine product (3a). In light of these findings, further study is on-going as regard the search for appropriate modification to achieving benzothiazepine products from these sulfonamides.

\section{CONFLICT OF INTEREST}

The authors hereby declare that there is no conflict of interest as regard this research article.

\section{ACKNOWLEDGEMENTS}

O.O.A. is grateful to the Academy of Science for the Developing World (TWAS) and Chinese Academy of Sciences (CAS) for the joint sponsorship of this work under the 2009 TWAS-CAS Postgraduate Fellowship Award.

\section{SUPPLEMENTARY MATERIAL}

Supplementary material is available on the publisher's web site along with the published article.

\section{REFERENCES}

[1] Eshghi, H.; Rahimizadeh, M.; Zokaei, M.; Eshghi, S.; Eshghi, S.; Faghihi, Z.; Tabasi, E.; Kihanyan, M. Synthesis and antimicrobial activity of some new macrocyclic bis-sulfonamides and disulfides. Eur. J. Chem., 2011, 2, 47-50.

[2] Connor, E.E. Sulfonamide antibiotics, Prim. Care Update Ob. Gyns., 1998, 5, 32-35.

[3] Ghosh, A.K. Capturing the essence of organic synthesis: From bioactive natural products to designed molecules in today's medicine. J. Org. Chem., 2010, 75, 7967-7989.

[4] Bertucci, A.; Innocenti, A.; Zoccola, D.; Scozzafava, A.; Tambutte', S.; Supuran, C.T. Carbonic anhydrase inhibitors. Inhibition studies of a coral secretory isoform by sulfonamides. Bioorg. Med. Chem., 2009, 17, 5054-5058.

[5] Bhat, M.A.; Imran, M.; Khan, S.A.; Siddiqui, N. Biological activities of sulfonamides. Indian J. Pharm. Sci., 2005, 67,151-159.

[6] Chohan, Z.H.; Shad, H.A.; Youssoufi, M.H.; Hadda, T.B. Some new biologically active metal-based sulfonamide. Eur. J. Med. Chem., 2010, 45, 2893-2901.

[7] Özbek, N.; Katircioğlu, H.; Karacan, N.; Baykal, T. Synthesis, characterization and antimicrobial activity of new aliphatic sulfonamide. Bioorg. Med. Chem., 2007, 15, 5105-5109.

[8] Basanagouda, M.; Shivashankar, K.; Kulkarni, M.V.; Rasal, V.P.; Patel, H.; Mutha, S.S.; Mohite, A.A. Synthesis and antimicrobial studies on novel sulfonamides containing 4-azidomethyl coumarin. Eur. J. Med. Chem., 2010, 45, 1151-1157.

[9] Warshakoon, H.J.; Burns, M.R.; David, S.A. Structure-activity relationships of antimicrobial and lipoteichoic acid-sequestering properties in polyamine sulfona-mides. Antimicrob. Agents Chemother., 2009, 53, 57-62.

[10] Johnson, D.C.; Widlanski, T.S. Cerium(iii) chloride-mediated reaction of sulfonamide dianions. J. Org. Chem., 2003, 68, 53005309.

[11] Bahrami, K.; Khodaei, M.M.; Soheilizad, M. Direct conversion of thiols to sulfonyl chlorides and sulfonamides. J. Org. Chem., 2009, 74, 9287-9291.

[12] Barbosa, M.L. C.; Melo, G. M.A.; Da Silva, Y.K. C.; Lopes, R.O.; De Souza, E.T.; De Queiroz, A.C.; Smaniotto, S.; AlexandreMoreira, M.S.; Barreiro, E.J.; Lima, L.M. Synthesis and pharmacological evaluation of $N$-phenylacetamide sul- fonamides. Eur. J. Med. Chem., 2009, 44, 3612-3620.

[13] Luca, L.D.; Giacomelli, G. An easy microwave-assisted synthesis of sulfonamides directly from sulfonic acids. J. Org. Chem., 2008, 73, 3967-3969.

[14] Steurer, M.; Bolm, C. Synthesis of amino-functionalized sulfonimidamides and their application in the enantioselective Henry reaction. J. Org. Chem., 2010, 75, 3301-3310.

[15] Meshram, G.A.; Patil, V.D. A simple and efficient method for sulfonylation of amines, alcohols and phenols with cupric oxide under mild conditions. Tetrahedron Lett., 2009, 50, 1117-11121.

[16] Argyropoulou, I.; Geronikaki, A.; Vicini, P.; Zanib, F. Synthesis and biological evaluation of sulfonamide thiazole and benzothiazole derivatives as antimicrobial agents. ARKIVOC, 2009, 6, 89100 .

[17] Da Silva, L.E. ; De Souza, P.T.; Maciel, E.N.; Nunes R.K.; Iriane, E.; Mario, S.; Rebelo, R.A. In vitro antiprotozoal evaluation of zinc and copper complexes based on sulfonamides containing 8aminoquinoline ligands. Lett. Drug Des. Discov., 2010, 7, 679-685. Da Silva, L.E.; De Sousa, P.T. Synthesis, structure and physicochemical properties of zinc and copper complexes based sulfona- 
mides containing 8-amino quino line ligands. Quim. Nova, 2008, 31, 1161-1164.

[19] Blank, B.R.; Alayoglu, P.; Engen, W.; Choi, J.K.; Berkman, C.E.; Anderson, M.O. N-Substituted glutamyl sulfonamides as inhibitors of glutamate carboxy peptidase II (GCP2). Chem. Biol. Drug Des., 2011, 77, 241-247.

[20] Lea, Z.G.; Chen, Z.C.; Hu, Y.; Zheng, Q.G. Organic Reactions in Ionic Liquids. A simple and highly regioselective $N$-substitution of pyrrole. Synthesis, 2004, 12, 1951-1954.

[21] Kim, J.G.; Jang, D.O. Mild and efficient indium metal catalyzed synthesis of sul-fonamides and sulfonic esters. Synlett, 2007, 16, 2501-2504.

[22] Chohan, Z.H.; Supuran, C.T. Structure and biological properties of first row d-transition metal complexes with $\mathrm{N}$-substituted sulfonamides. J. Enzyme Inhib. Med. Chem., 2008, 23, 240-251.

[23] Chohan, Z.H.; Shaikh, A.U.; Naseer, M.M.; Supuran, C.T. In-vitro antibacterial, antifungal and cytotoxicity properties of metal-based furanyl derived sulfonamides. J. Enzyme Inhib. Med. Chem., 2006, 21, 771-781.

[24] Chen, Y.T.; Xie, J.; Seto, C.T. Pepetidic ó-ketocarboxylic acids and sulfona mides as inhibitors of protein tyrosine phosphatases. $J$. Org. Chem., 2003, 68, 4123-4125.

[25] Maclean, D.; Hale, R.; Chen, M. The reversed kenner linker: A new safety-catch linker for the preparation of $N$-alkyl sulfonamide. Org. Lett., 2001, 3, 2977-2980.

[26] Wagman, A.S.; Wang, L.; Nuss, J.M. Simple and efficient synthesis of 3,4-di hydro-2-pyridones via novel solid-supported aza-annulation. J. Org. Chem., 2000, 65, 9103-9113.

[27] Yan, B.; Nguyen, N.; Liu, L.; Holland, G.; Raju, B. Kinetic comparison of trifluoroacetic acid cleavage reactions of resin-bound carbamates, ureas, secondary amides and sulfonamides from benzyl-, benzylhydryl-, and indole-based linkers. J. Comb. Chem., 2000, 2, 66-74.

[28] Hoye, R.C.; Richman, J.E.; Dantas, G.A.; Lightbourne, M.F.; Shinneman, L.S. Synthesis of polyazamacrocyclic compounds via Richman-Atkins cyclization of $\beta$-trimethylsilylethanesulfonamides. J. Org. Chem., 2001, 66, 2722-2725.

[29] Bisai, A. Prasad, B.A.B.; Singh, V.K. Aminolysis of $N$ tosylaziridines: An approach to asymmetric synthesis of symmetric and unsymmetric chiral sulfonamide ligands. ARKIVOC, 2007, 5, 20-37.

[30] Hu, J.B.; Zhao, G.; Yang, G-S.; Ding, Z.D. Asymmetric borane reduction of prochiral ketones by polymer-supported chiral sulfonamides. J. Org. Chem., 2001, 66, 303-304.

[31] Brouwer, A.J.; Liskamp, R.M.J. Synthesis of cyclic peptidosulfonamides by ring-closing metathesis. J. Org. Chem., 2004, 69, 3662-3668.
[32] Lopez, M.; Bornaghi, L.F.; Driguez, H.; Poulsen, S.A. Synthesis of sulfonamide- bridged glycomimetics. J. Org. Chem., 2011, 76, 2965-2975.

[33] Domínguez, L.; Nhien, A.N.V.; Tomassi, C.; Len, C.; Postel, D.; Marco-Contelles, J. Synthesis of 4-amino-5- $\mathrm{H}$-2,3-dihydroisothiazole-1,1-dioxide ring systems on sugar templates via carbanionmediated sulfonamide intramolecular cyclization reactions (CSIC Protocols) of glyco-ó-sulfonamidonitriles. J. Org. Chem., 2004, 69, 843-856.

[34] Kang, S.K.; Ko, B.S.; Ha, Y.H. Radical addition of p-toluenesulfonyl bromide and $p$-toluenesulfonyl iodide to allenic alcohols and sulfonamides in the presence of AIBN: Synthesis of heterocyclic compounds. J. Org. Chem., 2001, 66, 3630-3633.

[35] Lukin, O.; Schubert, D.; Müller, C.; Corda, M.; Kandre, R. Persulfonylation of amines applies to the synthesis of higher dendrimer. J. Org. Chem., 2008, 73, 3562-3665.

[36] Balan, D.; Adolfsson, H. Selective formation of $\alpha$-methylene- $\beta$ amino acid deri-vatives through aza version of the Baylis-Hillman reaction. J. Org. Chem., 2001, 66, 6498-6501.

[37] Ali, E.S.H.; Nassar, F.I.; Badawi, A.M.; Afify, S.H. Physical properties and biological applications of novel substituted biphenylsulfonamides. Int. J. Genet. Mol. Biol., 2010, 2, 78-91.

[38] Andrighetti-Fröhner, C.A.; De Oliveira, K.N.; Gaspar-Silva, D. Pacheco, L.K.; Joussef, A.C.; Steindel, M.; Simões, C.M.O.; De Souza, A.M.T.; Magalhaes, U.O.; Afonso, I.F.; Rodrigues, C.R.; Nunes, R.J.; Castro, H.C. Synthesis, biological evaluation and SAR of sulfonamide 4-methoxychalcone derivatives with potential antileishmanial activity. Eur. J. Med. Chem., 2009, 44, 755-763.

[39] Al-Soud, Y.A.; Al-Sa'doni, H.H.; Amjaour, H.A.S.; Salih, K.S.M.; Mubarak, M.S.; Al-Masoudi, N.A.; Jaber, I.H. Synthesis, characterization and anti-HIV and antitumor activities of new coumarin derivatives. Z. Naturforsch., $\mathrm{B}_{\mathrm{i}}, \mathbf{2 0 0 8}, 63 b, 83-89$.

[40] Milne, H.B.; Peng, C.H. The use of benzylsulfonyl chloride in peptide syntheses J. Am. Chem. Soc., 1957, 79, 639-644.

[41] Bakker, W.I.I.; Familoni, O.B.; Padfield, J.; Snieckus, V. Intramolecular anionic Friedel-Crafts equivalents. An expeditious synthesis of 4H-1,2-benzothiazin-4-one 1,1-dioxide from $\mathrm{N}$ arylsulfonylated amino acid. Synlett., 1997, 1997, 1079-1080.

[42] Wardencki, W.; Curyło, J.; Namieśnik, J. Green chemistry-present and future issues. Pol. J. Environ. Stud., 2005, 14, 389-395.

[43] Zhang, W.; Qin, Y.; Zhang, S.; Luo, M. Synthesis of novel Nheterocyclic car bene-Rh complexes derived from L-proline and their catalysis in the addition of arylboronic acids to aldehyde. ARKIVOC, 2005, 14, 39-48.

[44] Kuethe, J.T.; Beutner, G.L. Synthesis of 2-arylindole-4-carboxylic amides: [2-(4-fluorophenyl)-1H-indol-4-yl]-1-pyrrolidinylmethanone. Org. Synth., 2009, 86, 92-104.

(C) Ajani et al.; Licensee Bentham Open.

This is an open access article licensed under the terms of the Creative Commons Attribution Non-Commercial License (http://creativecommons.org/licenses/ by-nc/3.0/) which permits unrestricted, non-commercial use, distribution and reproduction in any medium, provided the work is properly cited. 